\title{
Seyfert Activity and Nuclear Star Formation in the Circinus Galaxy
}

\author{
R. Maiolino ${ }^{1,2}$, A. Krabbe ${ }^{1,3}$, N. Thatte ${ }^{1}$, R. Genzel ${ }^{1}$
}

\begin{abstract}
We present high angular resolution $\left(0.15^{\prime \prime}-0.5^{\prime \prime}\right)$ near infrared images and spectroscopy of the Circinus galaxy, the closest Seyfert 2 galaxy known. The data reveal a non-stellar nuclear source at $2.2 \mu \mathrm{m}$ whose size is smaller than $1.5 \mathrm{pc}$ in radius. The coronal line region and the hot molecular gas emission extend for $20-50 \mathrm{pc}$ in the ionization cone. The data do not show evidence for a point-like concentration of dark mass; we set an upper limit of $4 \times 10^{6} M_{\odot}$ to the mass of a putative black hole.

We find evidence for a young nuclear stellar population, with typical ages between $4 \times 10^{7}$ and $1.5 \times 10^{8}$ yrs. The luminosity of the starburst inside a few hundred pc is comparable to the intrinsic luminosity of the Seyfert nucleus, and the two of them together account for most of the observed bolometric luminosity of the galaxy. Within the central $12 \mathrm{pc}$ the starburst has an age of about $7 \times 10^{7}$ yrs and radiates $\sim 2 \%$ of the luminosity of the active nucleus. We discuss the implications of these results for models that have been proposed for the starburst-AGN connection.
\end{abstract}

Subject headings: galaxies: individual (Circinus) — galaxies: active — galaxies: starburst — galaxies: kinematics and dynamics — galaxies: nuclei — infrared: galaxies

\section{Introduction}

The connection between starburst and Seyfert activity is one of the most hotly debated issues in the context of AGNs. A variety of data have provided growing evidence that

\footnotetext{
${ }^{1}$ Max-Plank-Institut für Extraterrestrische Physik, Postfach 1603, D-85740 Garching, Germany

${ }^{2}$ Osservatorio Astrofisico di Arcetri, L.go E.Fermi 5, I-50125 Firenze, Italy

${ }^{3}$ DLR-Institute for Space Sensor Technology, Rudower Chaussee 5, 12489 Berlin, Germany
} 
starburst and Seyfert phenomenon often coexist (Cid Fernandes \& Terlevich 1995, Delgado and Perez 1993, Genzel et al. 1995, Heckman et al. 1989, 1995, Maiolino et al. 1995, Moorwood et al. 1996c, Neff et al. 1994, Oliva et al. 1995, Rodriguez-Espinosa et al. 1987, Storchi-Bergmann et al. 1996, Cid Fernandes et al. 1997). However, most of these studies have detected star forming activity, and/or a young stellar population in Seyfert galaxies (especially type 2) on the kpc scale, which might not be directly relevant to the active nucleus. Indeed, Maiolino et al. (1997) speculate that star formation in the host galaxy of Seyfert 2 nuclei is probably related only indirectly to the active nucleus: non-axisymmetric morphologies (bars/distortions/interactions) are likely to be the prime cause of the relationship, as they both enhance star formation in the galaxy and drive gas into the nuclear region to obscure the active nucleus. Terlevich and collaborators (Terlevich \& Melnick 1985, Terlevich et al. 1992, 1995, Terlevich 1994) have proposed a much closer link between nuclear star formation and Seyfert activity. They claim that the whole phenomenology observed in AGN can be accounted for by assuming that these nuclei are powered by a massive nuclear starburst evolving in the metal rich environment of early type galaxies: the ionizing radiation emitted by an extreme population of Wolf-Rayet stars, along with fast shocks generated by SNe, would produce the spectrum observed in Sy galaxies. So far, very few observational tests of this theory have been made, due to difficulties in probing star formation and stellar populations in the innermost region of AGNs. However, recently Heckman et al. (1997) have discovered a powerful, young (in the Wolf-Rayet phase) nuclear starburst in the Sy2 galaxy Mkn477; such a nuclear starburst might dominate the bolometric luminosity of the central region, therefore partly supporting Terlevich's scenario, at least in this object. Norman \& Scoville (1988), following Bailey(1980) and David (1987a,b), have proposed a different link between the nuclear stellar population and AGN. According to their model, a young nuclear ( $\leq 10 \mathrm{pc})$ star cluster would return mass into the interstellar medium, by means of post-main-sequence stars, that would feed a nuclear black hole. An analogous model has been proposed more recently by Murphy et al. (1991), who also include effects of tidial disruption of stars close to the black hole. Jenkins \& Binney (1994) have proposed a similar mechanism for the fueling of the Galactic center. Again, these models have not been tested, so far, due to the shortage of observations that can probe the nuclear stellar population.

The Circinus galaxy is a nearby $\left(4 \mathrm{Mpc}\right.$ ), edge-on (incl. $\left.\sim 65^{\circ}\right), \mathrm{Sb}-\mathrm{d}$ system that is seen through a low interstellar extinction window near the Galactic plane $\left(A_{V}=1.5 \mathrm{mag}\right.$, Freeman et al. 1977). The nuclear optical line ratios (Oliva et al. 1994) are typical of a Seyfert 2 galaxy. The Seyfert 2 classification is supported also by the detection of intense coronal lines (Oliva et al. 1994, Moorwood et al. 1996a), the discovery of an intense $\mathrm{X}$-ray iron $6.7 \mathrm{keV}$ line (Matt et al. 1996), rapid variation of the powerful $\mathrm{H}_{2} \mathrm{O}$ maser 
emission (Greenhill et al. 1997) and a prominent ionization cone in the [OIII] $\lambda 5007$ maps (Marconi et al. 1995), with filamentary supersonic outflows (Veilleux \& Bland-Hawthorn 1997). The large equivalent width of the Fe $6.7 \mathrm{keV}$ line detected by ASCA (EW $\sim 2 \mathrm{keV})$ indicates that the nucleus is heavily absorbed along the line of sight: $N_{H}>10^{25} \mathrm{~cm}^{-2}$, i.e it is Compton thick. The Circinus galaxy is also characterized by enhanced star forming activity: $\mathrm{H} \alpha$ and [SII] narrow band images (Marconi et al. 1995) have revealed the presence of a star forming ring at a radius of $\sim 10^{\prime \prime}=200 \mathrm{pc}$. Given its proximity and the coexistence of both star formation and an active nucleus, the Circinus galaxy appears to be one of the most suitable objects to tackle the starburst-AGN connection issue.

In this paper we present high angular resolution near-IR images and integral field spectroscopic data that probe the physics of the nuclear region of the Circinus galaxy, on scales from a few parsecs to $\sim 100$ pc. Using these data, we investigate the properties of the active nucleus and its interaction with the circumnuclear environment, with emphasis on the connection between Seyfert and stellar activity.

\section{Observations}

\subsection{D integral field spectroscopy}

The nucleus of the Circinus galaxy was observed in March and April 1996 with 3D, the MPE near-IR imaging spectrometer (Weitzel et al. 1996), assisted by ROGUE, a first order adaptive optics system (Thatte et al. 1995), at the 2.2m MPE-ESO telescope. 3D slices the focal plane into 16 slits and disperses their light in wavelength; the spectrum of the slices is then imaged onto a NICMOS3 detector $(256 \times 256$ pixels $)$. The resulting "cube" provides simultaneous spectra of an area $4.8^{\prime \prime} \times 4.8^{\prime \prime}$, as projected on the sky, divided into $16 \times 16$ pixels (i.e. $0.3^{\prime \prime} / \mathrm{pix}$ ). We observed Circinus in two different spectral configurations: in March (two nights) we observed the whole $\mathrm{K}$ band at a spectral resolution of $\mathrm{R}=1000$, while in April (four nights) we observed the spectral region around the CO stellar bands $(2.2-2.4 \mu \mathrm{m})$ at a spectral resolution of $\mathrm{R}=2000$. The FWHM of the point spread function (PSF) during the observations, after the tip tilt correction, was about $0.5^{\prime \prime}-0.6^{\prime \prime}$ in the $\mathrm{K}$ band. One of the nights in April had an optical seeing of $0.4^{\prime \prime}$, while the seeing in the $\mathrm{K}$ band, after tip tilt correction, was about $0.35^{\prime \prime}$, though we could not determine it accurately because of heavy undersampling of the PSF. We observed a nearby $\mathrm{O}$ star to correct the atmospheric transmission features in the $\mathrm{R}=1000$ data, as $\mathrm{O}$ stars are almost featureless in the $\mathrm{K}$ band, while we observed an A star to correct the $\mathrm{R}=2000$ data, as A stars are featureless in the corresponding spectral region. Data reduction was performed as described in Weitzel et al. (1996) and Thatte et al. (1997). 


\subsection{Speckle observations}

We also observed the Circinus galaxy by means of SHARP, the MPE speckle near-IR imaging camera at the NTT ESO telescope, in April 1996. This camera uses a NICMOS3 array with a pixel scale of $0.05^{\prime \prime} /$ pix (providing a field of view of $12.8^{\prime \prime} \times 12.8^{\prime \prime}$ ). We observed the central region of Circinus in $\mathrm{H}$ and $\mathrm{K}$ bands at a frame rate of $1 \mathrm{~Hz}$. The observations of Circinus were interleaved with observations of a reference star (about the same magnitude as the Circinus nucleus) to monitor the PSF. The individual images were background subtracted, flat fielded, shifted to center the brightest speckle and then co-added. Details on the data reduction are given in Eckart et al. (1993, 1995). The K band images have a PSF of 0.2" FWHM; however, if the diffuse, seeing limited PSF is subtracted, the central spike is close to the diffraction limit $\left(\sim 0.15^{\prime \prime} \mathrm{FWHM}\right)$. In $\mathrm{H}$, the PSF is slightly worse: $0.27^{\prime \prime}$ FWHM.

\section{Results}

This section is aimed at showing general results extracted from the data, to provide an overview of the features observed in the central region of the Circinus galaxy. A more detailed analysis is given in Sect. 4 \& 5 .

\subsection{Near-IR images}

Fig.1a shows the K-band SHARP image of the central region of Circinus, smoothed with a 3 pixel $\left(0.15^{\prime \prime}\right)$ FWHM gaussian to improve the $\mathrm{S} / \mathrm{N}$ on the low surface brightness features; while Fig.1b shows the central $3^{\prime \prime}$ at full resolution. The $\mathrm{K}$ band image shows a two armed spiral-like morphology. The western arm is also apparent in the $7000 \AA$ image (Marconi et al. 1995), but the southern arm is less evident in the optical image, which might be partly due to obscuration towards the South-East (see below) and partly to lack of angular resolution in the optical images. In the central region $(\mathrm{R} \leq 80 \mathrm{pc})$ the surface brightness distribution is smoother and rises slowly toward the $\mathrm{K}$ peak, with a shallow power law (surface brightness $\propto R^{-\alpha}$ with $\alpha \sim 0.5-0.7$ ). This central region $(\mathrm{R} \leq 80 \mathrm{pc}$ ) bay be considered as the inner part of a bulge (a "nuclear bulge", in analogy with our Galaxy, Mezger et al. 1996), though in this galaxy an outer bulge seems to be absent or very faint. The nuclear $\mathrm{K}$ band spike is unresolved and is most likely due to emission by hot dust, as we will discuss in sect.4.1. The hot dust emission might also affect the $\mathrm{H}$ band nuclear peak, though this is more difficult to test. 
Fig. 2 shows the $\mathrm{H}-\mathrm{K}$ color map. To generate such a map we had to center the $\mathrm{K}$ band image relative to the $\mathrm{H}$ image. Since the nucleus might be heavily reddened, the peak of the $\mathrm{H}$ light might be offset with respect to the $\mathrm{K}$ peak. Therefore, we did not determine the relative position of the two maps by aligning their peaks. Instead, we used both the cross-correlation of the two maps (excluding the peaks) and the location of a point source situated north of the nucleus. The $\mathrm{H}-\mathrm{K}$ color of an unreddened stellar population does not change significantly as it evolves; indeed, both spiral and ellipticals show a fairly narrow distribution of the $\mathrm{H}-\mathrm{K}$ color, which clusters at 0.22 mags, with a standard deviation of about 0.1 mag (Frogel 1985, Giovanardi \& Hunt 1996, Hunt et al. 1996). As a consequence, the $\mathrm{H}-\mathrm{K}$ excess relative to the standard value traces regions affected by reddening. This is not the case for the Seyfert nucleus, because it has intrinsically redder colors. Moreover, PSF differences between $\mathrm{H}$ and $\mathrm{K}$ make color gradients around the nuclear spike unreliable. However, that is not a problem for the extended emission, where small variations of the PSF do not significantly affect the colors. Fig.2 indicates a color gradient towards the SE. It is unlikely that this gradient is an instrumental effect due to imperfect flat fielding, as we found the same result by using different types of flat fields (night sky, twilight, and even without any flat field). Such a gradient very likely reflects an increase in the extinction towards the SE; this variation of the reddening is expected, as the SE is the near side of the galactic disk and therefore the dusty screen is expected to be more effective in this region (Quillen et al. 1995). However, the most interesting feature is the dust lane that enters the nuclear region from East toward South and twists toward the nucleus by 90 degrees at about $80 \mathrm{pc}$ from the center. Such a dust lane $\left(A_{V} \simeq 7.8\right)$ can account almost entirely for the interarm depression near the southern spiral arm observed in the K image (Fig.1a), though a stellar spiral component is probably also present.

\subsection{Spectroscopic data: emission lines distribution}

In Fig.1a the solid line box indicates the region sampled by $3 \mathrm{D}$ during the $\mathrm{R}=1000$ observations. Fig.3a shows the $3 \mathrm{D}, \mathrm{R}=1000$, spectrum extracted from an aperture of $0^{\prime \prime} .75$ (=15pc) centered on the nucleus. The spectrum has been rebinned to $\mathrm{R} \sim 700$ to improve the signal to noise. Most prominent in this spectrum are the coronal lines: [SiVI] (ionization energy $167 \mathrm{eV})$ f, [CaVIII] $(128 \mathrm{eV})^{4}$, and [AlIX] $(285 \mathrm{eV})^{4}$; the latter is the first detection in an extragalactic object. Tab.1 lists the fluxes of the lines detected in the nuclear region. The continuum rising toward longer wavelengths, and the stellar features

\footnotetext{
${ }^{4}$ This is the ionization energy of the lower ionization stage, i.e. the minimum energy required to obtain the observed ion.
} 
being much shallower than in normal galaxies, indicates that the non-stellar emission from the active nucleus contributes significantly to the continuum, diluting the stellar features. We extracted line maps by interpolating the underlying continuum with a first order fit in the $\Delta \lambda \sim 0.2 \mu \mathrm{m}$ around each line (by excluding regions affected by other lines and stellar features). An accurate continuum subtraction is one of the most critical issues, as the continuum slope and shape changes significantly over the field of view. Fig.4 shows maps of some of the lines detected in the $\mathrm{K}$ band spectrum. The last contour in each map is at $3 \sigma$ above the noise. The [SiVI] map extends in the direction of the ionization cone traced by the [OIII] maps, as indicated by means of yellow dashed lines (Marconi et al. 1995, Veilleux \& Bland-Hawtorn 1997). In contrast, the $\operatorname{Br} \gamma$ emission is more diffuse and extends also along the major axis of the galaxy (P.A. 25 $5^{\circ}$ see note 5$)$. The [AlIX] shows the most compact emission. The nuclear $\mathrm{H}_{2}$ emission is elongated towards the West, i.e. along the ionization cone, as [SiVI] does, but at lower surface brightness levels it also extends towards the NE, along with the $\operatorname{Br} \gamma$ emission. We will discuss these line maps in sections 4 and 5 .

\subsection{Spectroscopic data: stellar kinematics}

The dashed rectangle in Fig.1a indicates the region of the nucleus sampled by the 3D observations at $R=2000$. Fig. $3 b$ shows the $R=2000$ nuclear spectrum, extracted from an aperture of $0^{\prime \prime} .75$. For comparison, the $3 \mathrm{D}$ spectrum of a template star (M3I) is shown in Fig.3c (note the expanded scale on the $y$ axis).

We have used such spectra to map the stellar velocity field in the nuclear region of the Circinus galaxy. Both the galaxy spectra and the stellar template spectra were continuum subtracted. Then we used two different methods to determine both the velocity of the stellar system and its velocity dispersion. We first used a method similar to that described in Tonry \& Davis (1979): the cross-correlation of the galaxy spectrum with the template star was fitted by the autocorrelation function of the star convolved with a gaussian, whose $\sigma$ and peak velocity were free parameters. The second method consisted of fitting the galaxy spectrum in real space with a template stellar spectrum convolved with a gaussian: in this case the fit parameters were the $\sigma$ of the gaussian profile, the velocity shift applied to the stellar spectrum, and a multiplicative factor to fit the depth of the stellar features. Fitting was performed by using different stellar templates ( $\mathrm{K}-\mathrm{M}$ giants and supergiants) to monitor possible uncertainties introduced by a mismatch between the stellar template and

the observed galaxy's stellar population. To minimize these mismatch effects, when working in real space, we separately fitted each stellar feature, so that the minimization of the $\chi^{2}$ was not limited by differences in the relative depths of the features between the stellar 
template and the galaxy spectrum. Both fitting methods, i.e. fitting of the cross-correlation function and fitting in real space, gave consistent results. As the velocity dispersion turns out to be quite low $(\sim 80 \mathrm{~km} / \mathrm{s})$, i.e close to our resolution element, fitting the higher order moments instead of a simple gaussian (i.e. the kurtosis $h_{3}$ and $h_{4}$ ) was found not to be provide additional information when compared to uncertainties. Errors were estimated by determining the variation of the fit parameters that decreased the $\chi^{2}$ probability by $68 \%$. Not all the stellar features in the spectrum could be used: the ${ }^{12} \mathrm{CO}(3-1)$ band is corrupted by the [CaVIII] emission line; the ${ }^{13} \mathrm{CO}(2-0)$ band is affected by a helium emission line; the ${ }^{12} \mathrm{CO}(4-2)$ band is affected by some atmospheric features or by some unidentified emission line; the NaI doublet is corrupted by some instrumental effect in the nuclear spectrum (see Fig.3b), but could be used in other regions. Therefore, we used only the ${ }^{12} \mathrm{CO}(2-0)$ band and the CaI triplet over the whole field of view, and the NaI doublet only in the off nuclear regions (though the latter does not affect the results significantly).

Fig.5 shows the map of the stellar peak velocity as derived by fitting a cubic spline to a grid of 21 points obtained by rebinning the field of view of $3 \mathrm{D}$, to improve the signal-to-noise of each spectrum. The errorbars in the outer regions of the map are $\pm 15 \mathrm{~km} / \mathrm{s}$. Fig. 6 shows the stellar peak velocity and velocity dispersion along the major and minor axis of the galaxy (P.A. $25^{\circ}$ and $155^{\circ}$ respectively) as measured by means of the $3 \mathrm{D}$ spectra (filled dots). The stellar velocity dispersion is roughly constant in the central 100 pc. In Fig. 6 we also plot, with hollow squares, the peak velocity determined by using the [NII] emission line data from Oliva et al. (1994)․ The velocity field along the major axis shows a noticeable rotation pattern.

\section{The Seyfert nucleus}

\footnotetext{
${ }^{5}$ The major axis of the galaxy is subject to some uncertainty. Freeman et al. (1977) quote P.A. $30^{\circ}$. The RC3 catalog gives P.A. $40^{\circ}$. Elmouttie et al. (1995) give a P.A. $\sim 30^{\circ}$ based on an AAT optical image. The near-IR images in Marconi et al. (1995) should be less affected by extinction and light from the young stellar population than optical images are. The major axis of the isophotes of these IR images is at P.A. $\sim 25^{\circ}$. We suspect that the optical images are affected by patchy extinction in our Galactic plane, especially at the low surface brightness level. Indeed, the optical images show a weird tilt towards the East of the northern extremity of the galactic disk, which might be due to larger Galactic absorption in the northern region. Also, the crowding of Galactic sources makes it difficult to identify of the low surface brightness isophotes, probably used to measure the inclination of the galaxy. In this paper we assume the P.A. of the major axis to be that determined by means of the NIR images of Marconi et al. (1995), i.e. $25^{\circ}$. However, results do not change significantly if the actual major axis is $30^{\circ}$ as determined by Freeman et al. and Elmouttie et al..

${ }^{6}$ Their long slit was oriented almost in the same direction as the major axis: P.A. $20^{\circ}$.
} 


\subsection{The nuclear dust emission}

The shallow stellar features in the nuclear $\mathrm{K}$ band spectrum, the slope of the spectrum (Fig.3) and the extremely red colors of the nucleus indicate that the non-stellar emission from the Seyfert nucleus contributes significantly to the radiation observed in the nuclear region and that it dilutes the stellar features. As discussed by several authors (Efstathiou \& Rowan-Robinson 1995 EF95, Granato \& Danese 1994 GD94, Oliva et al. 1995, Thatte et al. 1997, Pier \& Krolik 1993 PK93, McAlary \& Rieke 1988), such K band radiation from Seyfert nuclei is very likely emitted by hot dust, at temperatures close to the sublimation limit $(\sim 1500 \mathrm{~K})$, heated by the intense UV radiation field emitted by the Seyfert source. As a consequence, the $\mathrm{K}$ band images cannot be used to trace the stellar light in the nuclear region. The $\mathrm{H}$ band nuclear peak is also likely to be affected by hot dust emission (Thatte et al. 1997, Oliva et al. 1995).

However, the CO stellar bands provide information about the fraction of the nuclear flux that is stellar. By assuming that the intrinsic equivalent width of the $\mathrm{CO}$ bands of the stellar population in the nuclear region is the same as in the circumnuclear region $(\mathrm{R}$ $\sim 50-70 \mathrm{pc}$ ), where the contribution of the nuclear hot dust source is absent, we can then use the measured EW(CO) on the nucleus to provide the amount of non-stellar dilution, or the fraction of the observed radiation that actually comes from stars. As the mass-to-light ratio turns out not to change significantly within the central $\sim 200$ pc (sect.5.2 and Fig.11), the intrinsic EW of the CO stellar bands is not expected to change much within the central region. Therefore, the fraction of the stellar light in the nuclear region can be reliably estimated in the way we just described, with an accuracy of about $20 \%$.

In Fig.7 we show the K-band surface brightness profile around the nuclear peak as derived from the SHARP image (filled dots and solid line), normalized to its peak intensity, compared with the fraction of stellar light (hollow circles) as derived by means of the CO

stellar bands. The innermost point for the stellar light fraction is determined by extracting the 3D spectrum from an aperture of $0^{\prime \prime} .3$ (6 pc) from the data of the night with best seeing $\left(\sim 0^{\prime \prime} .3\right)$. The dotted line indicates a fit to the stellar data using a modified King profile. It is noticeable that most of the nuclear emission is indeed non-stellar. If such a nuclear non-stellar source were unresolved, then the observed K-band profile should be the sum of the shallow stellar profile and a nuclear PSF, the latter being normalized to the peak value of the non stellar light (the PSF with this normalization is plotted with hollow squares connected by a long-dashed line). The sum of these components is indicated by the short-dashed line in Fig.7 and, within uncertainties, it is identical to the observed K profile. Therefore, the non-stellar $\mathrm{K}$-band source is point like at our resolution, i.e. it has a radius smaller than $1.5 \mathrm{pc}$. 
If Circinus were a type 1 Seyfert such a result would not be surprising, as the size of the region containing dust close to the sublimation limit is much smaller than 1 pc. Moorwood et al. (1996a) estimate an intrinsic luminosity of the Seyfert nucleus of $\sim 10^{10} L_{\odot}$, therefore, by assuming the grain mixtures given in Rowan-Robinson (1986), we derive a size of the hot $(1500 \mathrm{~K})$ dust region $R_{\text {sub }} \simeq 0.03 \mathrm{pc}$. In case of type 2 Seyferts the modelling is more complex. According to the unified model (Antonucci 1993) the nuclear hot dust should be partly obscured by the dense circumnuclear molecular torus. Therefore, the observed non-stellar near IR light should be partly associated with the short-wavelength tail of the mid-IR emission, and partly to radiation from the nuclear hot dust transmitted through the torus, the relative contribution of the two components being dependent on the optical thickness of the torus. Various authors have modelled the radiative transfer processes through the putative torus to determine the expected IR emission from type 2 Seyferts (PK93, GD94, ER95). In the model used by Granato et al. (1997) to fit the IR spectrum of NGC 1068, the size of the $2.2 \mu \mathrm{m}$ source is expected to be $\sim 70 \times R_{\text {sub }}$ (at a brightness level of $30 \%$ of the peak). If we scale the same model to Circinus, the size of the $2.2 \mu \mathrm{m}$ source is expected to be $\sim 1$ pc in radius, consistent with our upper limit. Siebenmorgen et al. (1997) have proposed a spherically symmetric model to specifically fit the IR emission from Circinus nucleus. However, their model predicts a size of 4-4.5 pc (in radius) for the emitting region in the near-IR: such a value is 3 times larger than our upper limit at $2.2 \mu \mathrm{m}$. Therefore, the axisymmetric geometry expected from the unified model better fits the observational constraints in terms of size of the emitting region in the near-IR.

In Tab.2 we compare the luminosity of the non stellar nuclear K component, as determined from our data, along with the luminosity in the $\mathrm{L}^{\prime}(3.8 \mu \mathrm{m}), \mathrm{M}(4.8 \mu \mathrm{m})$ and N $(10.3 \mu \mathrm{m})$ bands from Siebenmorgen et al. (1997); the latter data should be little affected by stellar light within the small aperture listed in Tab.2. The continuum rises steeply toward longer wavelengths. When compared to models, either PK93 or GD94 or ER95, such a steeply rising continuum indicates that the putative torus should be observed nearly

edge-on. More specifically, given that the light cone in Circinus is quite wide (opening angle $\sim 100^{\circ}$ ), ER95's model can reproduce the observed spectral energy distribution only if the torus is highly inclined, so that it can significantly self-absorb the near-IR emitting region.

\subsection{The coronal lines}

As discussed by several authors (Korista \& Ferland 1989, Oliva et al. 1994, Moorwood et al. 1996a, Oliva 1997, Marconi et al. 1996), the coronal lines observed in AGNs are most likely emitted by gas highly ionized by the hard UV-X ray nuclear radiation. Our [SiVI] 
map (Fig.4b), showing the coronal line emission extending into the narrow line region, supports such a picture. However, the scenario is more complex.

The [SiVI] $(167 \mathrm{eV})$ coronal line emission map extends preferentially in the western direction, up to $\sim 50 \mathrm{pc}$ from the nucleus. Instead, the emission region of the [AlIX] (285 $\mathrm{eV}$ ) line is much more compact (Fig.4c) and, if any, it is only barely resolved. This is better illustrated by Fig.8, where we plot the radial surface brightness of [SiVI], [AlIX] and of the PSF, within the sector $-60^{\circ} \leq P . A . \leq-95^{\circ}$. The $[$ AlIX] radial profile departs from the PSF only by $1 \sigma$, while [SiVI] is clearly resolved. Also, Fig. 8 indicates that the ionization state of the Narrow Line Region changes with radius in the western direction: at about $20 \mathrm{pc}$ the $[\mathrm{AlIX}] /[\mathrm{SiVI}]$ ratio is 2.7 times lower than on the nucleus at a confidence level of $5 \sigma$. The difference is less pronunced in the northern part of the ionization cone, where the $[\mathrm{SiVI}]$ surface brightness drops faster and whose radial profile is closer to that of $\left[\right.$ AlIX]. Possibly, the western part of the radiation cone (P.A. $\sim-90^{\circ}$ ) intercepts the dense, circumnuclear molecular clouds in the galactic plane, thereby lowering the ionization parameter lower[ and increasing the emissivity of the coronal lines $\left(\epsilon \propto n^{2}\right)$. This would explain the lower $[\mathrm{AlIX}] /[\mathrm{SiVI}]$ ratio in this region and the enhanced [SiVI] emission with respect to the northern part of the ionization cone. Another possibility is that the ionization mechanism is different in the western extension. Collisional ionization, due to fast shocks, could contribute in this region. However, Oliva (1997), Oliva et al. (1994) and Marconi et al. (1996) show that ionization of the coronal gas as a consequence of fast shocks is very unlikely in AGNs, and specifically in Circinus.

Finally, it is interesting to note that the peak of the [SiVI] emission is slightly shifted, by $0^{\prime \prime} .15$ (=3pc), with respect to the $\mathrm{K}$ band nucleus and to the peak of the $\operatorname{Br} \gamma$ and [AlIX] emission. Although higher resolution data are required to confirm such a finding, it is worthwhile to compare this result with the ionization structure expected to occur in the (inner) coronal line region (CLR). As discussed in Marconi et al. (1994), if the CLR is "thick" for $\mathrm{Si}^{+5}$, i.e. the $\mathrm{He}^{+}$continuum opacity is responsible for determining its ionization structure, the peak of the $[\mathrm{SiVI}]$ emission should occur at the $\mathrm{He}^{+2}$ Strömgren radius, i.e.

$$
R_{[S i V I](\text { peak })}=\left[\frac{Q\left(H e^{+}\right)}{n_{e}^{2} \alpha_{B}\left(H e^{+}\right)}\right]^{1 / 3}
$$

(assuming a filling factor close to unity). By using the parameters for the Circinus

\footnotetext{
${ }^{7}$ Oliva (1997) warns that the ionization parameter at the inner face of the cloud might be meaningless when dealing with the coronal line region (CLR), while a quasi-spherical photoionization model is more appropriate given the estimated pc-scale size of the CLR. However, at the distances (>20 pc) that we are considering here, the plane parallel slab model is a good enough approximation for the clouds in the galactic disk that are illuminated by the AGN continuum.
} 
CLR given in Moorwood et al. (1996a), i.e. $Q\left(H e^{+}\right) \simeq Q(H) / 2 \simeq 10^{53} \mathrm{phot} / \mathrm{sec}$ and

$n_{e} \simeq 5000 \mathrm{~cm}^{-3}$, we estimate $R_{[S i V I](\text { peak })} \sim 5 \mathrm{pc}$, in agreement with the valuie observed in the 3D map. Such gas, responsible for the nuclear coronal emission lines, must intercept only a fraction of the nuclear solid angle, allowing the UV-X radiation to ionize gas in the outer light cone.

A more detailed discussion on the coronal emission lines, including the [CaVIII] line (which requires a careful subtraction of the underlying stellar features) and their kinematical properties, will be presented in a forthcoming paper.

\subsection{The molecular gas}

There are three main mechanisms that may be responsible for the emission of the $\mathrm{H}_{2}$ lines observed in the near IR. These lines can be excited thermally in hot gas, either heated by shocks (Hollenbach \& Shull 1977, Draine 1980, Draine et al. 1983) or by intense X-ray radiation (Lepp \& McCray 1983, Maloney \& Hollenbach 1996, Tiné et al. 1997), or can be emitted under fluorescent excitation conditions if the UV radiation field is strong enough (Black \& van Dishoeck 1988, Sternberg 1988). Near IR spectroscopy of AGN and starburst galaxies has indicated that UV fluorescence pumping is unlikely to play a major role in most of these objects (Moorwood \& Oliva 1990, Sugai et al. 1997), and therefore thermal excitation must prevail. However, Sternberg \& Dalgarno (1989), have studied the UV excitation with a more detailed model and found that $i$ ) the UV field can contribute to the gas heating and $\mathrm{ii}$ ) when the gas densities are higher than $10^{4} \mathrm{~cm}^{-3}$ collisional de-excitation of the $\mathrm{H}_{2}$ levels becomes important, and the emitted spectrum resembles the thermal case.

The maps of the $\mathrm{H}_{2}$ emission from the Circinus galaxy (Figs.4.d-e) indicate that the nuclear emission is elongated 10-20 pc towards the West, i.e. in the same direction as the $\mathrm{X}$-ray excited [SiVI] line. However, this is not sufficient evidence to support $\mathrm{X}$-ray heating, as the UV field in this direction is also intense. Moreover, at lower surface brightness levels the $\mathrm{H}_{2}$ emission also extends in the NE direction, i.e. it extends in the same direction as the $\operatorname{Br} \gamma$ emission ( $\propto$ UV radiation), which probably traces star forming activity (sect.5.1).

The $\mathrm{H}_{2}$ line ratios help to better understand between various excitation mechanisms. The curves in the diagrams on the left side of Fig.9 show the expected fluxes of some of the $\mathrm{H}_{2}$ lines, relative to $\mathrm{H}_{2}(1-0) \mathrm{S}(1)$, as a function of temperature, for pure thermal excitation. The dots in the diagrams on the right side show the same ratios according to the combined UV pumping+thermal models of Sternberg \& Dalgarno (1989), as a function of gas density, for their $\chi$ parameter (proportional to the intensity of the UV radiation) ranging from 10 
to $10^{4}$; except for the case of $(1-0) \mathrm{Q}(1)$, for which only the case $\chi=10^{2}$ is given in their paper.

The hatched areas indicate the ratios observed on the nucleus, and their thickness give the $\pm 1 \sigma$ range. The lightly-shaded areas indicate the ratios observed in an aperture $\sim$ $20 \mathrm{pc} \mathrm{NE}$ of the nucleus. The observed $(2-1) \mathrm{S}(1) /(1-0) \mathrm{S}(1)$ ratio clearly rules out pure UV pumping (the low density regime of Sternberg \& Dalgarno's models) as the excitation mechanism in both regions. Thermal excitation fits the data of the $\mathrm{H}_{2}$ nuclear emission, within $\sim 1.2 \sigma$, if the gas has a temperature of about $2500 \mathrm{~K}$. For the UV+thermal models the agreement with the nuclear line ratios is worse, this is because in the very high density, quasi-thermal regime the molecular gas reaches temperatures that are too low $(\sim 1000 \mathrm{~K})$. However, we cannot exclude that by varying the parameters in Sternberg \& Dalgarno's model a better agreement can be obtained. The similar western extension of the $\mathrm{H}_{2}$ and [SiVI] lines, together with these results suggests that the nuclear $\mathrm{H}_{2}$ lines are mostly emitted thermally in gas heated by the X-ray, and possibly UV, radiation emitted by the AGN. If this were also the case for other AGNs (e.g. NGC4945, Moorwood et al. 1996b), then the $\mathrm{H}_{2}$ emission line distribution in active nuclei would not necessarily trace the distribution of molecular gas, but it would also reflect the distribution of the $\mathrm{X}$-ray radiation field.

In the NE off-nuclear region, where extended $\operatorname{Br} \gamma$ emission is seen, the thermal model reproduces poorly the observed $\mathrm{H}_{2}$ line intensities, as different line ratios measure different temperatures. In this region UV pumping might contribute to the gas excitation. More specifically, Sternberg \& Dalgarno's model could fit the observed ratios if the gas density is close to the transition between UV-fluorescence and thermal regimes, i.e. $\sim 10^{4.5} \mathrm{~cm}^{-3}$.

\subsection{The nuclear dynamical mass}

As illustrated in Fig.6, within the central 100 pc the bulge is isothermal within uncertainties. The smallest aperture over which we can sample the velocity dispersion is given by the seeing during the observations, i.e. $\sim 0^{\prime \prime} .5-0^{\prime \prime} .6(=10-12 \mathrm{pc})$. Though on one night the seeing was as good as $\sim 0^{\prime \prime} .3$, data from that night alone do not have a high enough signal-to-noise to sample the stellar velocity dispersion within the innermost $0^{\prime \prime} .3$, also due to higher dilution from the hot dust component in the central aperture. Following Kormendy (1988) and Kormendy \& Richstone (1995), we extracted the spectrum of the nuclear stellar cluster $(\mathrm{R} \leq 6 \mathrm{pc})$, cleaned of the bulge contribution, by subtracting the mean of the spectra at $\pm 17 \mathrm{pc}$ along the minor axis (which have the same velocity shift as the nucleus). The resulting stellar spectrum has a velocity dispersion of $55 \pm 15 \mathrm{~km} / \mathrm{s}$. The velocity dispersion is lower in the nuclear region indicating that we do not detect a nuclear 
point-like concentration of mass (Binney \& Tremaine, 1987). By assuming the nuclear stellar cluster to have a virialized, isotropic distribution and a King core radius of $10 \mathrm{pc}$, as derived from Fig.7, we estimate an upper limit of $4 \times 10^{6} M_{\odot}$ to any compact concentration of mass. The crucial point, as we will show in sect.5.2, is that such a nuclear mass is not associated with a mass-to-light ratio higher than that of the circumnuclear region, as would be expected in the case of a nuclear concentration of dark mass significant with respect to the stellar mass.

If the AGN is powered by an accreting black hole, then our upper limit on the black hole mass indicates that the $\mathrm{AGN}$ is radiating at a rate $\left(10^{10} L_{\odot}\right.$, Moorwood et al. 1996a) that is higher than 0.1 times its Eddington limit, i.e. $\mathrm{L}_{A G N} / \mathrm{L}_{E d d}>0.1$. In NGC 1068, another Compton thick Seyfert 2 nucleus, $\mathrm{L}_{A G N} / \mathrm{L}_{E d d}=0.2$ (Greenhill et al. 1996, Pier et al. 1994). Such high values of the $\mathrm{L} / \mathrm{L}_{E d d}$ ratio might be common in these highly obscured AGNs. Indeed, as pointed out by Granato et al. (1997), the $\mathrm{L} / \mathrm{L}_{E d d}$ ratio is expected to increase with the circumnuclear column density (in their model $\mathrm{L} / \mathrm{L}_{E d d} \propto N_{H}^{2}$ ).

\section{Star formation and stellar population}

As discussed in the introduction, the $\mathrm{H} \alpha$ and [SII] maps (Marconi et al. 1995) show evidence for a star forming ring at $\mathrm{R}=200 \mathrm{pc}$, however $\mathrm{H} \alpha$ and [SII] emission is also observed inside the ring. The 3D Br $\gamma$ map (Fig.4a) shows evidence for star forming activity in the close vicinity of the Seyfert nucleus: indeed, the $\operatorname{Br} \gamma$ emission extends outside the ionization cone, along the galaxy major axis, at 20-40 pc from the Seyfert nucleus.

In this section we discuss the properties of the star formation and of the stellar population in the nuclear region of Circinus. We constrain age and luminosity of the stellar population by comparing the observed $\operatorname{Br} \gamma$ equivalent width $(\mathrm{EW}(\mathrm{Br} \gamma))$ and mass-to-light ratio with values predicted by the stellar population synthesis code described in Sternberg \& Kovo (1997) 8 . We assume a Salpeter initial mass function between $1 \mathrm{M}_{\odot}$ and $60 \mathrm{M}_{\odot}$, and a broken power law for lower masses (Miller \& Scalo, 1977). The star formation history is assumed to be exponentially decaying:

$$
S F R \propto \exp \left(-t / t_{0}\right)
$$

When dealing with the nuclear stellar population $(\mathrm{R}<6 \mathrm{pc})$ we consider two different models. Model "A" assumes the AGN and the starburst to be two different entities, and

\footnotetext{
${ }^{8}$ This code uses the stellar evolutionary tracks of Meynet et al. (1994) for solar metallicity, though in Sect.5.3.3 we also consider the case of $Z=2 Z_{\odot}$.
} 
that the Br $\gamma$ emission in the NLR is accounted for by the AGN. Model "B" assumes that the NLR is mostly ionized by a nuclear starburst (Terlevich's theory).

\section{1. $\operatorname{Br} \gamma$ equivalent width}

The ratio between Lyman continuum luminosity $\left(L_{L y c}^{*}\right)$ and luminosity emitted in the $\mathrm{K}$ band $\left(L_{K}^{*}\right) \mathrm{g}$ by the stellar photospheres is a function of the ratio between the number of massive hot stars and number of giant-supergiant red stars. Therefore, $L_{L y c}^{*} / L_{K}^{*}$ provides information on the star formation history. $\mathrm{EW}(\operatorname{Br} \gamma) \propto L_{L y c}^{*} / L_{K}^{*}$ if:

i) there is no significant contribution from non-stellar sources to the ionizing photon flux;

ii) no ionizing photons escape out of the plane of the galaxy;

iii) there is no differential extinction (in the $\mathrm{K}$ band) between the HII regions and the red giant-supergiant population (in this case $\mathrm{EW}(\mathrm{Br} \gamma)$ is independent of foreground extinction);

$i v$ ) there is no contribution from non-stellar reprocessed light (e.g. hot dust emission) in the $\mathrm{K}$ band.

Fig.10a shows the evolution of $\mathrm{EW}(\mathrm{Br} \gamma)$ expected for continuous star formation $\left(t_{0}=10^{9} \mathrm{yrs}\right)$, an instantaneous burst $\left(t_{0}=10^{6} \mathrm{yr} s\right)$, and the intermediate case of $t_{0}=10^{7}$ yrs, as derived by means of Sternberg \& Kovo's code, and assuming that hypotheses $i-i v$ are true.

Within the central $\sim 20 \mathrm{pc}$, the Br $\gamma$ emission is dominated by the PSF of the innermost part of the Narrow Line Region (NLR). When considering model A, regions contaminated by the NLR must be avoided. The lightly shaded area in Fig.10a indicates the EW that we observe in the circumnuclear region, at 30-40 pc radius, outside the ionization cone (i.e. North and North-East). In this region we assume negligible deviations from conditions $i-i v$. The age of the burst derived from Fig.10a is naturally quite sensitive to the assumed length of the burst $\left(t_{0}\right)$.

When dealing with model $B$ (Terlevich's model) the $\operatorname{Br} \gamma$ emission from the NLR must be included in the parameters constraining the stellar population, i.e we should use the $\mathrm{EW}(\mathrm{Br} \gamma)$ measured on the nucleus $(\mathrm{R}<6 \mathrm{pc})$. However, as discussed in Sect.4.1, the

\footnotetext{
${ }^{9} \mathrm{~L}_{K}$ is defined as the luminosity emitted in the 1.9-2.5 $\mu \mathrm{m}$ spectral range.
} 
nuclear stellar light is significantly diluted by hot dust emission in the $\mathrm{K}$ band. Therefore, we corrected the nuclear $\mathrm{EW}(\operatorname{Br} \gamma)$ by subtracting the continuum contribution from hot dust. Moreover, the nuclear Lyman continuum flux is known to escape out of the plane of the galaxy through the ionization cone. Thus, we corrected for this effect by dividing the $\mathrm{EW}(\mathrm{Br} \gamma)$ by the covering factor. Moorwood et al. (1996a) estimate the nuclear covering factor to be 0.05 by comparing the Lyman continuum flux, as measured by means of the $\mathrm{H} \alpha$ flux from a spatially resolved cloud within the ionization cone, and the nuclear $\operatorname{Br} \alpha$ flux (dereddened according to the nuclear Balmer decrement). The hatched area in Fig.10a indicates the nuclear $(\mathrm{R}<6 \mathrm{pc}) \mathrm{EW}(\mathrm{Br} \gamma)$ corrected for hot dust emission and covering factor. We will discuss the effect of possible differential extinction in Sect.5.3.3.

\subsection{The mass-to-light ratio}

The mass-to-light ratio in the $\mathrm{K}$ band, $\mathrm{M} / \mathrm{L}_{K}^{*}$ (see note 9 ), of the stellar population can better constrain both the age of the stellar population and the length of the burst $t_{0}$.

The K-band (projected) stellar luminosity was determined for various apertures by means of the SHARP $\mathrm{K}$ image at radii $1^{\prime \prime} .5<R<5^{\prime \prime}$ and by using photometric data given in Moorwood \& Glass (1984) at larger radii. Similar to what is required for the $\mathrm{EW}(\operatorname{Br} \gamma)$, we are interested only in the $\mathrm{K}$ band light emitted directly by stellar photospheres and not in the contribution from reprocessed light. Therefore, when measuring $\mathrm{L}_{K}^{*}$ the contribution from the nuclear non stellar spike was subtracted. At radii $<1^{\prime \prime} .5$, we estimated the fraction of the K-luminosity that is stellar by means of the stellar CO map, as described in sect.4.1.

The extinction affecting the $\mathrm{K}$-band stellar light was estimated by means of the $\mathrm{H}-\mathrm{K}$ color excess with respect to the unreddened value of 0.22 (sect.3.1). By assuming the extinction law given in Koorneef (1983), a foreground screen model gives $A_{K}=1.4 \cdot E_{H-K}$. The estimated extinction ranges from an average value of $\mathrm{A}_{K}=0.3$, within $18^{\prime \prime}$, to $\mathrm{A}_{K}=0.63$ at 1."5. The latter value is consistent with that derived by Oliva et al. (1995) using stellar spectra in both $\mathrm{H}$ and $\mathrm{K}$ bands, and it is also close to the extinction $A_{K}=0.52$ derived for the innermost $\left(\sim 1^{\prime \prime}\right)$ part of the NLR by means of the hydrogen emission lines (Oliva et al. 1994). Colors involving shorter wavelength bands have larger intrinsic scatter amongst field galaxies and are more sensitive to age and metallicity effects, therefore they are less suitable to derive the $\mathrm{K}$-band extinction. However, it is possible to perform a consistency check by comparing the $\mathrm{V}-\mathrm{K}$ color with that expected for young and evolved stellar populations. Within the central $18^{\prime \prime} \mathrm{V}-\mathrm{K}=4.3$ (Moorwood \& Glass 1984), when compared to the average colors of ellipticals ( $\mathrm{V}-\mathrm{K} \sim 3.3$, Frogel et al. 1978) it yields $\mathrm{A}_{K} \sim 0.1$, while if compared to

intrinsic colors expected for young stellar populations $\left(\mathrm{V}-\mathrm{K} \sim 1.7\right.$ for a $10^{8}$ yrs old stellar 
population, Leitherer \& Heckman 1995) the observed $\mathrm{V}-\mathrm{K}$ color implies an $\mathrm{A}_{K}=0.3$. For apertures smaller than $\sim 1^{\prime \prime} .5$ the $\mathrm{H}-\mathrm{K}$ color cannot be used to derive the extinction $A_{K}$ because of the contribution from the Seyfert nucleus. Therefore, we assume the nuclear extinction to be the same as that derived from the $\mathrm{H}-\mathrm{K}$ color at $\sim 1^{\prime \prime} .5$. In sect.5.3.2 we will discuss the effects of nuclear extinction (within the nuclear $R<1^{\prime \prime} .5$ ) higher than that assumed here.

The included mass has been calculated by using the [NII] emission line data at radii $\geq 5^{\prime \prime}$, and assuming axisymmetric rotation. At radii $<2^{\prime \prime}$ we used the stellar velocity maps from 3D. We used the Boltzmann equation for a spherically symmetric space density distribution whose velocity dispersion is isotropic (Binney \& Tremaine, 1987). Initially, we have also assumed the mass tracers to have a constant mass-to-light ratio within the central region, in order to estimate the deprojected volume density of mass tracers from the observed surface brightness profile (such a volume density $\rho$ enters in the Jeans equation as a multiplicative factor in the form $\mathrm{d} \ln (\rho) / \mathrm{d} \ln (r))$. The latter bit of reasoning might sound circular, as we will use it to estimate the mass-to-light ratio of the stellar population; however, it is possible to iterate the procedure and, within uncertainties, check for self-consistency. Due to uncertainties both in the measurements and in the model, the errorbars range from $40 \%$ up to a factor of 2 of the estimated enclosed mass. The mass derived in such a way is the dynamical mass, which is actually an upper limit to the stellar mass. Indeed, the gas mass might contribute significantly to the dynamical mass. There are no CO millimetric interferometric measurements of the Circinus galaxy, but $\mathrm{CO}(2-1)$ data obtained with a $22^{\prime \prime}$ beam (Aalto et al. 1995) indicate that the gas mass enclosed within the star forming ring is about $6 \times 10^{8} M_{\odot}$, if the Galactic conversion factor is assumed (Kenney \& Young 1989), i.e. about half of the dynamical mass in the same region. On the other hand, the galactic conversion factor might not be appropriate in the central active region of the galaxy (Aalto et al. 1995, Casoli et al. 1992) and, therefore, the amount of molecular gas determined by means of the $\mathrm{CO}(2-1)$ line might be overestimated. Finally, the old stellar bulge population might contribute significantly to the dynamical mass, thus affecting the measure of mass-to-light ratio for the starbursting population.

Fig.11 shows the estimated mass-to-light ratio $\mathrm{M} / \mathrm{L}_{K}^{*}$ of the stellar population within various apertures. Though uncertainties are large, the $\mathrm{M} / \mathrm{L}_{K}^{*}$ is clearly larger outside the star forming ring than inside it, indicating a younger stellar population in the inner region. In the central $30 \mathrm{pc}$ the $\mathrm{M} / \mathrm{L}_{K}^{*}$ ratio increases slightly, probably due to the mass contribution from an older stellar population, though it is still sustantially lower than typical $\mathrm{M} / \mathrm{L}_{K}^{*}$ in bulges of normal galaxies and in ellipticals $(\sim 15 \text { and } \sim 35 \text { respectively })^{\text {to }}$

\footnotetext{
${ }^{10}$ These values are from Oliva et al. 1995, converted to our units, where we have deduced $\mathrm{L}_{K}$ from their
} 
characterized by a fairly old stellar population. In particular, we do not find a nuclear peak in the $\mathrm{M} / \mathrm{L}$ as would be expected from the presence of a massive dark object.

Fig.10b shows the expected $\mathrm{M} / \mathrm{L}_{K}^{*}$ ratio vs. age of the stellar population, as determined by using the Sternberg \& Kovo code, for the same burst models as in Fig.10a. The dark-shaded area corresponds to the $\mathrm{M} / \mathrm{L}_{K}^{*}$ determined just inside the star forming ring, the lightly-shaded area corresponds to the value determined within $40 \mathrm{pc}$ from the nucleus, while the hatched area gives the $\mathrm{M} / \mathrm{L}_{K}^{*}$ on the nucleus $(\mathrm{R} \leq 6 \mathrm{pc})$. For a given $\mathrm{M} / \mathrm{L}_{K}^{*}$ the age of the stellar population depends on the burst duration to a lesser extent than the $\mathrm{Br} \gamma$ equivalent width.

\subsection{Starburst models and implications for the Starburst-AGN connection}

\subsubsection{Starburst activity within $R<200$ pc (model A)}

We initially consider model A, i.e. the AGN and nuclear star formation are two different entities and the AGN accounts for the Br $\gamma$ emission in the NLR. By comparing Figs.10a and $\mathrm{b}$, we note that within the central $\mathrm{R} \leq 40 \mathrm{pc}$ (lightly shaded areas) the constant star formation model $\left(t_{0}=10^{9}\right)$ does not fit both observational constraints, i.e. $\mathrm{EW}(\mathrm{Br} \gamma)$ and $\mathrm{M} / \mathrm{L}_{K}^{*}$. In other words, in case of constant star formation the burst would have converted into stars a mass that is a factor of 100 higher than the observed dynamical mass. The $\delta$-burst model $\left(t_{0}=10^{6}\right)$ also fits poorly both $\mathrm{M} / \mathrm{L}_{K}^{*}$ and $\mathrm{EW}(\operatorname{Br} \gamma)$ constraints, though modelling of the $\delta$ burst is more uncertain as it is more sensitive to the discrete sampling of the stellar evolutionary tracks. The parameters that best fit the observations within the central $40 \mathrm{pc}$ are a moderate duration of the burst, $t_{0} \sim 10^{7} \mathrm{yrs}$, and an age of the stellar population of $5 \times 10^{7} \mathrm{yrs}$. Similar parameters are also typical of other starburst nuclei (e.g. Lutz et al. 1996, Rieke et al. 1993).

If we assume that the same burst model $\left(t_{0}=10^{7} \mathrm{yrs}\right)$ applies to the whole region within the central $400 \mathrm{pc}$, then the observed distribution of M/L $\mathrm{L}_{K}^{*}$ (Fig.11) indicates that the age of the stellar population within this region ranges from $4 \times 10^{7}$ yrs to $1.5 \times 10^{8}$ yrs.

It is interesting to note that by including the star forming ring, i.e. the annulus at 200 pc traced by $\mathrm{H} \alpha$ and [SII] (Marconi et al. 1995), the M/L $\mathrm{L}_{K}^{*}$ increases by about a factor of 2 (Fig.11). This might indicate that such a ring has undergone a more recent burst and that it has not yet entered in the red-supergiants phase. Circinus could be experiencing an outward

$\mathrm{L}_{H}$ by assuming $\mathrm{H}-\mathrm{K}=0.22$. 
propagating starburst: the nuclear region $(\mathrm{R} \leq 100-150 \mathrm{pc})$ experienced a burst about $0.4-1.5 \times 10^{8}$ yrs ago, and its near-IR continuum is now dominated by red supergiants, while the starburst activity has propagated to $R \sim 200$ pc where it is currently forming young hot stars. Such a phenomenology is common to other starburst galaxies, such as M82 and IC342, where evidence has been found for a nuclear stellar population dominated by red supergiants few times $10^{7}$ yrs old, while a circumnuclear ring is undergoing a more recent burst that is producing young hot stars (Rieke et al. 1980, Satyapal et al. 1997, Förster et al. 1996, Böker et al. 1996, 1997).

Fig.10c shows the evolution of the bolometric-to-K band luminosity ratio, $L_{b o l}^{*} / L_{K}^{*}$, as a function of age, for the same models as in Figs.10a and b. Given an average age of $\sim 10^{8}$ yrs for the stellar population inside $\mathrm{R}=200 \mathrm{pc}$, we get $L_{b o l}^{*} / L_{K}^{*} \simeq 50$ for the $t_{0}=10^{7}$ yrs model. Note that the derived $L_{b o l}^{*} / L_{K}^{*}$ ratio is not very sensitive to the adopted burst model $\left(t_{0}\right)$, if the $\mathrm{M} / \mathrm{L}_{K}^{*}$ is used as the age indicator, as the various models in Fig.10b and Fig.10c have opposite trends versus age. In the extreme case of constant star formation (which we ruled out) the $L_{b o l}^{*} / L_{K}^{*}$ ratio increases only by a factor of 1.5 , while a $\delta$-burst would give the same $L_{b o l}^{*} / L_{K}^{*}$ ratio as the $t_{0}=10^{7}$ yrs model.

The stellar bolometric luminosity can be derived from the observed K-band stellar luminosity by using the following relation:

$$
L_{b o l}^{*}=\left(\frac{L_{b o l}^{*}}{L_{K}^{*}}\right)_{\text {model }} \times L_{K}^{*}(o b s)
$$

where $L_{K}^{*}(o b s)$ is the observed $\mathrm{K}$-band stellar luminosity corrected for extinction.

Given the $\mathrm{K}$ band luminosity within $\mathrm{R} \leq 200 \mathrm{pc}$, the total bolometric luminosity emitted by the stellar population within the same aperture is $L_{b o l}^{*} \sim 1.1 \times 10^{10} L_{\odot}$. This luminosity has to be compared with the intrinsic AGN luminosity, $10^{10} L_{\odot}$ (Moorwood et al. 1996a), and with the bolometric luminosity of the galaxy, $\sim 1.7 \times 10^{10} L_{\odot}$ (corrected for Galactic absorption, but not for internal extinction)巴, i.e. Seyfert nucleus and starburst contribute to a similar extent to the total bolometric luminosity. Tab.3 summarizes the luminosity budget of the various sources in Circinus. It is not surprising that the sum of the starburst and AGN luminosities is slightly larger than the observed bolometric luminosity: in this edge- on system a fraction of the light escapes perpendicular to the galactic plane and, therefore, is not reprocessed into IR radiation nor is directly observed; in particular, a

\footnotetext{
${ }^{11}$ The IR luminosity from IRAS data is $1.2 \times 10^{10} L_{\odot}$ (Siebenmorgen et al. 1997). By using the photometric data in Moorwood \& Glass (1983) along with the $\mathrm{B}_{T}$ in the RC3 catalog, corrected for a Galactic extinction $A_{V}=1.5$, we estimate a luminosity of $\sim 5 \times 10^{9} L_{\odot}$ in the $\mathrm{K}$ to $\mathrm{U}$ wavelength range.
} 
significant fraction of the radiation from the AGN is probably lost through its light cones. From the energetics point of view Circinus is similar to Mrk 477, the most powerful Seyfert 2 in the local Universe, for which Heckman et al. (1997) estimate that the nuclear (few 100 pc) starburst and the Seyfert nucleus provide most of the total luminosity of the galaxy, in about equal proportions. However, the starburst in Mrk 477 is at a much earlier stage (6 Myr) than the starburst in Circinus.

\subsubsection{Starburst activity within $R<6$ pc (model $A)$}

In the innermost $12 \mathrm{pc}$ the $\mathrm{Br} \gamma$ emission is affected by the emission from the NLR. If we assume that the intrinsic $\mathrm{EW}(\operatorname{Br} \gamma)$ of the starburst within the central $12 \mathrm{pc}$ is the same as that measured at $\sim 30-40 \mathrm{pc}$, then the nuclear $\mathrm{M} / \mathrm{L}_{K}^{*}$ ratio implies an age of the nuclear star cluster of about $7( \pm 3) \times 10^{7} \mathrm{yrs}$, and a burst length $t_{0}=10^{7} \mathrm{yrs}$.

The $L_{b o l}^{*} / L_{K}^{*}$ derived for this starburst model (Fig.10c) implies a bolometric luminosity (eq.1) of the nuclear stellar population of $\mathrm{L}_{b o l}^{*}(\mathrm{R} \leq 6 \mathrm{pc})=2( \pm 0.6) \times 10^{8} L_{\odot}$. Therefore, the nuclear stellar luminosity accounts for only $\sim 2 \%$ of the Seyfert luminosity estimated by Moorwood et al. (1996a).

As discussed in the introduction, David (1987b), Norman \& Scoville (1988) and Murphy et al. (1991) proposed models that ascribe the AGN fuelling to mass loss from post-main sequence stars. The gas ejected from post-main sequence red giants and supergiants is kinematically hot, as its velocity dispersion reflects the velocity dispersion of bulge stars ( $\sim 80 \mathrm{~km} / \mathrm{s}$ for Circinus, Fig.6), and therefore it is characterized by the large turbulent viscosity that is required for the gas to inflow. More specifically, Norman \& Scoville's (1988) model predicts the ratio between the luminosity of the active nucleus and the luminosity of the evolving stellar cluster, within the central $10 \mathrm{pc}$, to be $\sim 50$ when the nuclear cluster is 70 Myrs old. This number is in fair agreement with the ratio between the intrinsic Seyfert luminosity and the luminosity derived for the star cluster in the nuclear $12 \mathrm{pc}$ of Circinus: $L_{A G N} / L_{*}(R \leq 6 p c) \approx 46$. This result does not necessarily prove these models to be correct, but it is the first time that observational data provide a consistency check for the theory of AGN fuelling through stellar mass loss. Finally, we note that Thatte et al. (1997) and Oliva et al. (1995) have found, though on larger scales, that other Seyfert nuclei are characterized by similar moderately young stellar populations (few $10^{8} \mathrm{yrs}$ ). It is tempting to speculate that such intermediate age stellar populations are closely linked to Seyfert activity in the way we just depicted.

The working hypothesis of this model is that the extinction in the $\mathrm{K}$ band toward 
the nucleus is the same as that estimated by means of the H-K color at $30 \mathrm{pc}$ from the nucleus (i.e. $A_{K}(n u c)=0.63 \mathrm{mag}$, sect.5.2). However, the nucleus might be affected by an extinction higher than in the circumnuclear $30 \mathrm{pc}$. Correcting the nuclear $\mathrm{K}$ band luminosity for higher extinction results in a lower $\mathrm{M} / \mathrm{L}_{K}^{*}$ ratio, i.e. a younger stellar population. The $\mathrm{M} / \mathrm{L}_{K}^{*}$ ratio cannot be lower than the minimum value predicted by the model (Fig.10b), this constrains the maximum possible excess nuclear extinction to be $\Delta A=21.5 \mathrm{mag}$. If $\mathrm{L}_{K}^{*}(\mathrm{nuc})$ is corrected for excess nuclear extinction, then the estimated bolometric luminosity of the stellar population, $\mathrm{L}_{b o l}^{*}$, increases both because of the increased $\mathrm{L}_{b o l}^{*} / \mathrm{L}_{K}^{*}$ derived from the model (as the lower $\mathrm{M} / \mathrm{L}_{K}^{*}$ indicates a younger population) and because of the increased $\mathrm{L}_{K}^{*}$ after correcting for extinction (eq.1). Fig.12 indicates that if the extinction toward the nucleus is higher than what we derived from the circumnuclear region, the nuclear stellar cluster could be younger (as young as $10^{7} \mathrm{yrs}$ ) and more luminous (as luminous as $\mathrm{L}_{b o l}^{*}=2 \times 10^{9} L_{\odot}$ ). With regard to the implications for Norman \& Scoville's model, a younger star cluster would provide an even larger mass loss available to fuel the accreting black hole.

\subsubsection{Starburst activity within $R<6$ pc (model B)}

In model B (Terlevich's model) most of the luminosity of the active nucleus is due to starburst activity. In this case, as discussed in sect.5.1, the nuclear starburst also contributes to the ionization of the Narrow Line Region. Therefore, we should use the nuclear $\mathrm{EW}(\mathrm{Br} \gamma)$, corrected as described in sect.5.1 (hatched area in Fig.10a), to model the nuclear stellar population. A $5 \times 10^{6}$ yrs old $\delta$-burst fits both the $\mathrm{EW}(\mathrm{Br} \gamma)$ and $\mathrm{M} / \mathrm{L}_{K}^{*}$ ratio. The age derived for the nuclear star cluster is close to that expected in Terlevich's model during the Sy2 phase. However, the derived bolometric luminosity is $\sim 20 \times 10^{8} L_{\odot}$, which is only $20 \%$ of the luminosity of the Seyfert nucleus

However, the main problem is not the luminosity deficit, but the Spectral Energy Distribution (SED). Our starburst model predicts a SED peaking close to the Lyman edge, while the AGN radiates most of its energy around $100 \mathrm{eV}$ and in the hard $\mathrm{X}$-rays (Moorwood et al. 1996a). On the other hand, our model does not take into account the emission from SNR radiative shocks, expected to play a role at high energies in Terlevich's model.

\footnotetext{
${ }^{12}$ We should mention that the starburst models were also run by assuming the metallicity to be twice the solar value, to account for Terlevich's claim that AGN-like starbursts should be evolving in a metal rich environment, and the results were found not to change significantly.
} 
There are other factors that might affect our estimate of the bolometric luminosity of the nuclear star cluster. If the foreground extinction is not uniform, but the nuclear star cluster is more obscured than the Narrow Line Region (differential extinction), then the observed $\mathrm{EW}(\operatorname{Br} \gamma)$ is higher than that expected from $\mathrm{L}_{L y c}^{*} / \mathrm{L}_{K}^{*}$. In this case, the extinction-corrected $\mathrm{EW}(\mathrm{Br} \gamma)$ is lower than the observed value and the derived age of the burst is older, as shown in Fig.13. The corrected $\mathrm{M} / \mathrm{L}_{K}^{*}$ ratio cannot be lower than that expected by the model, and this constrains the maximum differential extinction to be $\mathrm{A}_{V}$ (stars) $-\mathrm{A}_{V}(\mathrm{NLR})<30 \mathrm{mag}$. The derived bolometric luminosity changes as a consequence of two competitive factors: $i) \mathrm{L}_{K}^{*}$ (corrected) increases, but ii) $\left(L_{b o l}^{*} / L_{K}^{*}\right)_{\text {model }}$ decreases as a consequence of the older age. The net behaviour is an increase of $\mathrm{L}_{b o l}^{*}$, as shown in Fig.13. The corrected $\mathrm{L}_{b o l}^{*}$ could be as high as $7 \times 10^{9} L_{\odot}$, for a differential extinction of 30 mags.

Moorwood et al. (1996a) estimate the Lyman continuum luminosity by using the $\mathrm{H} \alpha$ flux from a "resolved" cloud in the NLR, whose projected distance from the nucleus is known. However, there are a few factors which lead to an underestimate of the real $\mathrm{L}_{L y c}$ : i) a fraction of the $\mathrm{H} \alpha$ flux from the cloud might be lost as a consequence of dust extinction within the cloud, ii) a fraction of the ionizing photon flux might be absorbed by dust in front of or within the cloud, iii) the distance of the cloud from the nucleus might be larger than the observed projection, iv) the cloud might be made up of subclumps or filaments, i.e. the effective area of the cloud might be lower than observed. If the real $\mathrm{L}_{L y c}$ is higher than that estimated by Moorwood et al. (1996a), then in sect.5.1 we have undercorrected the nuclear $\mathrm{EW}(\mathrm{Br} \gamma)$ when accounting for the covering factor. Therefore, correcting the $\mathrm{L}_{L y c}$ for effects $i-i v$ makes the nuclear $\mathrm{EW}(\mathrm{Br} \gamma)$ larger and makes the derived age for the cluster younger, as shown in Fig.14. The maximum value of the $\mathrm{EW}(\operatorname{Br} \gamma)$ predicted by the model imposes a maximum correction to the Lyman continuum luminosity: $\mathrm{L}_{L y c}($ corrected $) / \mathrm{L}_{L y c}($ observed $)<13$. Correcting $\mathrm{L}_{L y c}$ affects both the derived $\mathrm{L}_{b o l}^{*}$ (because of the younger age of the cluster) and the bolometric luminosity of the AGN (because the total $\mathrm{L}_{L y c}$ is the normalizing factor of the SED derived by Moorwood et al. 1996a). The net effect is a decrease of the ratio $\mathrm{L}_{b o l}^{*} / \mathrm{L}_{b o l}(\mathrm{AGN})$. The most extreme correction would imply a very young nuclear cluster $\left(\sim 10^{6} \mathrm{yrs}\right)$ which radiates about $8 \%$ of the luminosity of the Seyfert nucleus.

Summarizing, if we assume that a compact nuclear starburst is responsible for most of the $\operatorname{Br} \gamma$ emission of the Seyfert nucleus, the starburst must be as young as $5 \times 10^{6}$ yrs, in fair agreement with the expectation of Terlevich's model, but the bolometric luminosity of the starburst is only $20 \%$ of the observed luminosity of the active nucleus. However, if the nucleus is affected by differential extinction the luminosity derived for the nuclear starburst could be higher by up to a factoor of 4 . Yet, the main problem of fitting the observed SED 
in Circinus at high energies with the softer emission expected from the nuclear starburst remains unsolved.

Finally, it is interesting to note that Veilleux \& Bland-Hawthorn (1997) estimated that the filamentary ejecta, extending radially from the nucleus of Circinus, were probably expelled by an explosive nuclear event that occurred a few Myr ago. Such a time scale fits with the age of the nuclear burst estimated in this section.

\section{Summary}

We have studied the nuclear region of the Circinus galaxy by means of near IR images which have an angular resolution $\simeq 0^{\prime \prime} .15$ ( $=3 \mathrm{pc}$ as projected on the source), and K-band integral field spectroscopy with an angular resolution $\sim 0^{\prime \prime} .5$ and spectral resolution of 1000 and 2000.

The K-band image reveals a nuclear non-stellar source that is unresolved, i.e. whose size is smaller than $1.5 \mathrm{pc}$ in radius. Such nuclear non-stellar light is most likely emitted by dust heated by the Seyfert nucleus. When compared to models of the IR emission from obscuring torii, our upper limit fits the expected size of the K-band emission in Sy2s.

The Coronal Line Region traced by the $[\mathrm{SiVI}]$ line extends for $\approx 50 \mathrm{pc}$ into the Narrow Line Region, but the higher excitation [AlIX] emission line is much more compact. The variation of the $[\mathrm{AlIX}] /[\mathrm{SiVI}]$ ratio in the light cone might indicate that the ionization parameter of the NLR clouds decreases radially due to photoionization of high density clouds in the galactic plane.

The nuclear emission from the $\mathrm{H}_{2}$ lines observed in the K-band extends in the same direction as the [SiVI] emission. The $\mathrm{H}_{2}$ line ratios indicate that such lines are emitted by thermally excited gas, probably heated by the same $\mathrm{X}$-ray radiation that excites [SiVI]. At lower surface brightness levels the $\mathrm{H}_{2}$ emission extends along the plane of the galaxy: in these regions UV-fluorescence might contribute to the excitation mechanism.

The stellar velocity field traced by the stellar features in the K-band does not show evidence for a point-like mass concentration in the nuclear region. Also, there are no hints of an increase in the mass-to-light ratio toward the nucleus. We set an upper limit of $4 \times 10^{6} M_{\odot}$ for the mass of a putative black hole. As a consequence, the $\mathrm{L}_{A G N} / \mathrm{L}_{E d d}$ ratio must be larger than 0.1 .

The $\operatorname{Br} \gamma$ map indicates evidence for ongoing star forming activity within a few tens of pc from the active nucleus. The mass-to-light ratio, from the $100 \mathrm{pc}$ scale to the innermost 
$10 \mathrm{pc}$, is quite low and indicates that the stellar population is relatively young, with an age ranging between $4 \times 10^{7}$ and $1.5 \times 10^{8} \mathrm{yrs}$. There are indications of an outward propagation of the starburst. The bolometric luminosity derived for the young stellar population inside the central $400 \mathrm{pc}$ is comparable to the (intrinsic) luminosity of the active nucleus, and both of them together contribute most of the bolometric luminosity of the galaxy. The stellar population within $\mathrm{R}<6 \mathrm{pc}$ has an age of $7 \times 10^{7} \mathrm{yrs}$ and contributes only $2 \%$ of the intrinsic luminosity of the active nucleus.

We have discussed models that ascribe AGN fuelling to mass loss from a young nuclear star cluster in the light of our observational constraints. Our results fit predictions of these models in terms of expected nuclear stellar luminosity relative to the AGN luminosity.

We have also considered the case of a nuclear starburst contributing significantly to the $\operatorname{Br} \gamma$ emission in the Narrow Line Region (Terlevich's hypothesis). In this case the nuclear $(\sim 10 \mathrm{pc})$ stellar population could be as young as $5 \times 10^{6} \mathrm{yrs}$, but the implied bolometric luminosity of the nuclear star cluster would be only $20 \%$ of the intrinsic luminosity of the active nucleus. However, differential extinction effects could lead to an underestimate of the luminosity of the nuclear star cluster by up to a factor of 4 .

We appreciate comments and helpful discussions with A. Moorwood, T. StorchiBergmann and G.L.Granato. We are grateful to S. Anders, A. Eckart, J. Gallimore and H. Kroker for help during the observations. We thank A. Sternberg for making available his code. R.M. acknowledge the support of the ASI grant ASI-95-RS-120. 


\section{REFERENCES}

Aalto, S., Booth, R.S., Black, J.H., \& Johansson, L.E.B., 1995, A\&A, 300, 369

Antonucci, R., 1993, ARA\&A, 31, 473

Bailey, M.E., 1980, MNRAS 191, 195

Binney, J.J, Tremaine, S., 1987, Galactic Dynamics, Princeton Univ. Press, Princeton, NJ

Black, J.H., van Dishoeck, E.F., 1987, ApJ, 322, 412

Böker, T., Förster, N., Krabbe, A., Genzel, R., 1996, in From Stars to Galaxies, ASP Conf. Ser. Vol. 98, Eds. C. Leitherer, U. Frits-v. Alvensleben, and J. Hucra, p.455

Böker, T., Förster, N., Genzel, R., 1997, ApJ submitted

Casoli, F., Dupraz, C., Combes, F., 1992, A\&A, 264, 55

Cid Fernandes, R., \& Terlevich, R., 1995, MNRAS 272, 423

Cid Fernandes, R., Schmitt, Storchi-Bergmann, T., 1997, MNRAS, submitt.

David, L.P., Durisen, R.H., Cohn, H.N., 1987a, ApJ 313, 556

David, L.P., Durisen, R.H., Cohn, H.N., 1987b, ApJ 316, 505

Delgado, R.M.G., Perez, E., 1993, Astroph. Sp. Sci 205, 127

Draine, B.T., 1980, ApJ, 241, 1021

Draine, B.T., Roberge, W.G., \& Dalgarno, A., 1983, ApJ, 264, 485

Eckart, A., Genzel, R., Hofmann, R., Sams, B.J., Tacconi-Garman, L.E., ApJ, 407, L77

Eckart, A., Genzel, R., Hofmann, R., Sams, B.J., Tacconi-Garman, L.E., ApJ, 445, L26

Efstathiou, A, Rowan-Robinson, M., 1995, MNRAS, 273, 649 (ER95)

Elmouttie, M., et al., 1995, MNRAS, 275, L53

Förster, N., Böker, T., Krabbe, A., Genzel, R., 1996, in From Stars to Galaxies, ASP Conf. Ser. Vol. 98, Eds. C. Leitherer, U. Frits-v. Alvensleben, and J. Hucra, p.417

Freeman, C., Karlsson, B., Lyng, G., Burrell, J.F., van Woerden, H., Goss, W.M., Mebold, U., 1977, A\&A, 55, 445 
Frogel, J.A., Persson, S.E., Matthews, K., Aaronson, M., 1978, ApJ, 220, 75

Frogel, J.A., 1985, ApJ, 298, 528

Genzel, R., et al., 1995, ApJ 444, 129

Giovanardi, C., Hunt, L.K., 1996, ApJ

Granato, G.L., Danese, L., 1994, MNRAS, 268, 235 (GD94)

Granato, G.L., Danese, L., Franceschini, A., 1997, ApJ, in press

Greenhill, L.J., Gwinn, C.R., Antonucci, R., Barvainis, R., 1996, ApJ 172, L21

Greenhill, L.J., Ellingsen, S.P., Norris, R.P., Gough, R.G., Sinclair, M.W., Moran, J.M., Mushotsky, R., 1997, ApJ 474, L103

Heckman, T.M., Blitz, L., Wilson, A.S., Armus, L., \& Miley, G.K. 1989, ApJ, 342, 735

Heckman, T.M., et al., 1995, ApJ 452, 549

Heckman, T.M., et al., 1997, ApJ, in press

Hollenbach, D.J, \& Shull, J.M., 1977, ApJ 216, 419

Hunt, 1.K., et al., 1997, ApJS, in press

Jenkins, A., Binney, J., 1994, MNRAS 270, 703

Kenney, J.D., \& Young, J.S., 1989, ApJ, 344, 171

Koornneef, J., 1983, A\&A, 128, 84

Korista, K.T., Ferland, G.J., 1989, ApJ, 343, 678

Kormendy, J., 1988, ApJ, 335, 40

Kormendy, J., Richstone, D., 1995, ARA\&A, 33, 581

Leitherer, C., Heckman, T.M., 1995, ApJS, 96, 9

Lepp, S., \& McCray, R., 1983, ApJ, 269, 560

Lin, D.N.C., Pringle, J.E., 1987, MNRAS, 225, 607

Lutz, D., et al., 1996, A\&A, 315, L137 
Maiolino, R., Ruiz., M., Rieke, G.H., Keller, L.D., 1995, ApJ 446, 561

Maiolino, R., Ruiz., M., Rieke, G.H., Papadopoulos, P., 1997, ApJ, 485, 522

Maloney, P.R., Hollenbach, D.J., Tielens, A.G.G.M., 1996, ApJ, 472, 582

Marconi, A., Moorwood, A.F.M., Salvati, M., \& Oliva, 1994, A\&A, 291, 18

Marconi, A., Moorwood, A.F.M., Origlia, L., Oliva, E., 1995, ESO Mess, 78, 20

Marconi, A., van der Werf, P.P., Moorwood, A.F.M., Oliva, E., 1996, A\&A,

Matt, G., et al., 1996, MNRAS, 208, 253

McAlary, C.W., Rieke, G.H., 1988, ApJ, 333, 1

Meynet, G., Meader, A., Schaller, G., Schaerer, D., \& Charbonnel, C., 1994, A\&AS, 103, 97

Moorwood, A.F.M., \& Oliva, E., 1990, A\&A, 329, 78

Moorwood, A.F.M., \& Glass, I.S. 1984, A\&A 135, 281

Moorwood, A.F.M., et al., 1996a, A\&A, 315, L109

Moorwood, A.F.M., van der Werf, P.P., Kotilainen, J.K., Marconi, A., Oliva, E., 1996b, A\&A, 308, L1

Moorwood, A.F.M., Origlia, L., Kotilainen, J.K., and Oliva, E., 1996c, in Proceedings of the ESO Workshop on Spiral Galaxies in the near IR, eds. D.Minniti and H.-W.Rix, p. 299

Murphy, B.W., Cohn, H.N., Durisen, R.H., 1991, ApJ, 370, 60

Neff, S., et al., 1994, ApJ 430, 545

Norman, C., Scoville, N., 1988, ApJ 332, 124 (NS)

Oliva, E., Salvati, M., Moorwood, A.F.M., Marconi, A., 1994, A\&A, 288, 457

Oliva, E., Origlia, L., Kotilainen, J.K., Moorwood, A.F.M., 1995, A\&A, 301, 55

Oliva, E., 1997, in Emission Lines in Active Galaxies: New Methods and Techniques, B.M.Peterson, F.-Z., Cheng, and A.S.Wilson (eds.), p.228

Pier, E.A., Krolik, J.H., 1993, ApJ, 418, 673 (PK93) 
Pier, E.A., et al., 1994, ApJ, 428, 124

Quillen, A.C., Frogel, J.A., Kuchinski, L.E., Terndrup, D.M., 1995, AJ, 110, 205

Rieke, G.H., Lebofsky, M.J., Thompson, R.I., Low, F.J., Tokunaga, A.T., 1980, ApJ 238, 24

Rieke, G.H., Loken, K., Rieke, M.J., Tamblyn, P., 1993, ApJ, 412, 111

Rodriguez-Espinosa, J., Rudy, R., Jones, B., 1987, ApJ 312, 555

Rowan-Robinson, M., 1986, MNRAS, 219, 737

Satyapal et al. 1997, ApJ submitted

Siebenmorgen, R., Moorwood, A., Freudling, W., Käufl, H-U., A\& A, in press

Sternberg, A., 1988, ApJ, 332, 400

Sternberg, A., Dalgarno, A., 1989, ApJ 338, 197

Sternberg, A., Kovo, 0., 1997, in preparation

Storchi-Bergmann, T., Rodriguez-Ardila, A., Schmitt, H.R., Wilson, A.S. Baldwin, J.A., 1996, ApJ, 472, 83

Suchkov, A.A., et al., 1994, ApJ, 430, 511

Sugai, H., Malkan, M.A., Ward, M., Davies, R.I., 1997, ApJ, 481, in press

Terlevich, R., Melnick, J., 1985, MNRAS 213, 841

Terlevich, R., Tenorio-Tagle, G., Franco, J., and Melnick, J., 1992, MNRAS 255, 713

Terlevich, R., 1994, in Violent Star Formation from 30 Doradus to QSOs, ed. G. Tenorio-Tagle, p. 329 MNRAS 255, 713

Terlevich, R., Tenorio-Tagle, G., Rozycka, M., Franco, J., and Melnick, J., 1995, MNRAS 272, 198

Thatte, N., Kroker, H., Weitzel, L., Tacconi-Garman, L.E., Tecza, M., Krabbe, A., Genzel, R., 1995, Proceedings of the SPIE, 2475, 228

Thatte, N., et al., 1997, ApJ, in press

Tiné, S., Lepp, S., Gredel, R., Dalgarno, A., 1997, ApJ, in press 
Tonry, J., Davis, M., 1979, AJ, 84, 1511

Veilleux, S., Bland-Hawtorn, J., 1997, ApJ, in press

Weitzel, L., Krabbe, A., Kroker, H., Thatte, N., Tacconi-Garman, L.E., Cameron, M., Genzel, R., 1996, A\&AS, 119, 531 
Fig. 1. - a) K-band SHARP image, smoothed with a 3 pixel $\left(=0.15^{\prime \prime}\right)$ FWHM gaussian. Contours give $\log \left(\mathrm{F}_{\lambda}\right)$, in units of $10^{-24} \mathrm{~W} \mathrm{~cm}^{-2} \mu \mathrm{m}^{-1} \operatorname{arcsec}^{-1}$, at the following levels: 0.6, $0.75,0.8,0.9,1.0,1.1,1.2,1.3,1.4,1.5,1.6,1.7,1.8$. The green solid-line and dashed-line boxes show the regions observed with $3 \mathrm{D}$ at a spectral resolution of $\mathrm{R}=1000$ and $\mathrm{R}=2000$ respectively. b) Enlargement of the central $3^{\prime \prime}$ of the unsmoothed K-band SHARP image. Contours are in the same units as in Fig.1a at the levels: 1.2, 1.3, 1.4, 1.5, 1.6, 1.8, 2.0, 2.2, 2.4 .

Fig. 2.- H-K color map, smoothed as Fig.1a. Contours are at 0.25, 0.35, 0.45, 0.55, 0.65, 0.75 mags. Regions affected by low signal-to-noise have been blanked.

Fig. 3.- a) Nuclear spectrum of Circinus, extracted from an aperture of $0.75^{\prime \prime}$, rebinned to a spectral resolution of 700 . Labels marked with a "*" show the position of absorption stellar features, while other labels identify emission lines. b) The long wavelength part of the nuclear spectrum (same aperture as $a$ ) at a spectral resolution of 2000. c) Spectrum of one of the template stars (HD94613, shifted to the redshift of Circinus) used to fit the profile of the stellar features in Circinus' spectra.

Fig. 4. - Maps of emission lines in the $\mathrm{K}$ band spectrum. The cross marks the position of the continuum peak. The last contour in each map is at $3 \sigma$ above the noise. a) $\operatorname{Br} \gamma$; contours are at $80 \%, 60 \%, 40 \%, 30 \%, 20 \%, 15 \%$ and $10 \%$ of the peak. $b$ ) [SiVI] (the yellow dashed lines indicate the position of the ionization cone traced by the [OIII] line maps from Marconi et al. 1995 and Veilleux \& Bland-Hawthorn 1997); contours are at 90\%,60\%, 40\% $, 30 \%, 20 \%, 15 \%, 7.5 \%$ and $5 \%$ of the peak. c) [AlIX]; contours are at $80 \%, 60 \%, 40 \%$, $30 \%, 20 \%, 17 \%$ and $12 \%$ of the peak. d) $\mathrm{H}_{2}(1-0) \mathrm{S}(1)$; contours are at $80 \%, 60 \%, 40 \%$, $30 \%, 20 \%, 15 \%$ of the peak. e) $\mathrm{H}_{2}(1-0) \mathrm{S}(2)$; contours are at $80 \%, 60 \%, 50 \%, 40 \%, 28 \%$ of the peak. In $d$ and $e$ two areas affected by bad pixels have been blanked.

Fig. 5. - Map of the stellar velocity field as measured by means of the IR stellar features in the 3D spectra. Contour levels are spaced by $10 \mathrm{~km} / \mathrm{s}$, from 224 to $294 \mathrm{~km} / \mathrm{s}$. Errorbars in the outer parts of the map are $\pm 15 \mathrm{~km} / \mathrm{s}$.

Fig. 6. - Stellar peak velocity and velocity dispersion as measured by means of the stellar features in the 3D spectra (filled dots). Hollow squares indicate the velocity derived by means of the $[\mathrm{NII}]$ emission line data in Oliva et al. (1994).

Fig. 7.- Radial profile of the nuclear K-band surface brightness as derived from the SHARP image (filled circles and solid line). Hollow circles indicate the fraction of the $\mathrm{K}$-band light emitted by stars as determined by means of the 3D maps of the CO stellar bands. The dotted line indicates a fit of the stellar fluxes by using a modified King profile. The hollow 
squares connected by a long dashed line indicated the PSF profile normalized to the peak of the non stellar light. The short-dashed line is the sum of the stellar component (dotted line) and of the PSF (long-dashed line).

Fig. 8.- Radial profile of the surface brightness of the [SiVI] and [AlIX] emission lines, compared to the PSF, in the sector $-60^{\circ} \leq P . A . \leq-95^{\circ}$.

Fig. 9.- Flux of some of the $\mathrm{H}_{2}$ lines detected in the $\mathrm{K}$ band relative to the $\mathrm{H}_{2}(1-0) \mathrm{S}(1)$ line. The hatched areas indicate the values observed on the nucleus ( $0.6^{\prime \prime}$ aperture), while the lightly-shaded areas indicate the ratios observed in an aperture at about $20 \mathrm{pc}$ NE of the nucleus. The thickness of the shaded areas gives the $\pm \sigma$ range. The curves in the diagrams on the left side indicate the ratios expected in case of emission from thermally excited gas, as a function of temperature. The dots in the diagrams on the right side give the ratios predicted by Sternberg \& Dalgarno's models (see text), as a function of gas density, for their parameter $\chi$ (that is proportional to the UV radiation field) ranging from 10 to $10^{4}$; except for the $(1-0) \mathrm{Q}(1)$ line where only the $\chi=10^{2}$ case is given.

Fig. 10. - a) Expected quivalent width of the Br $\gamma$ versus age of a starburst in case of a $\delta$ burst (dotted line), a burst duration of $10^{7}$ yrs (solid line) and continuous star formation (dashed line). The lightly-shaded area indicates the observed range of $\mathrm{EW}(\mathrm{Br} \gamma)$ at 30-40 pc from the nucleus, outside the ionization cone. The hatched area indicates the nuclear $\mathrm{EW}(\mathrm{Br} \gamma)$ after correcting the $\mathrm{Br} \gamma$ flux for the covering factor of the NLR and the K band light for contamination by hot dust. b) Expected mass-to-light ratio for the same models as in $a$. The darkly-shaded area gives the $\mathrm{M} / \mathrm{L}_{K}^{*}$ determined inside the star forming ring, the lightly-shaded area gives the same ratio inside $\mathrm{R}=40 \mathrm{pc}$, while the hatched area gives the $\mathrm{M} / \mathrm{L}_{K}$ on the nucleus $(\mathrm{R} \leq 6 \mathrm{pc})$. The solid-line and dashed-line boxes indicate the range of values observed in bulges of spirals and in ellipticals (from data in Oliva et al. 1995). c) Expected bolometric-to-K band luminosity for the same models as in $a$.

Fig. 11. - Mass-to-light ratio within various regions centered on the nucleus.

Fig. 12.- Effects of correcting the nuclear $\mathrm{K}$-band light for excess nuclear extinction with respect to the circumnuclear region, in model A. Top, effect on the age derived for the nuclear star cluster. Bottom, effect on the bolometric luminosity derived for the nuclear star cluster. See text about estimates of the maximum excess extinction.

Fig. 13. - Effects of correcting the nuclear $\mathrm{K}$-band light and $\mathrm{EW}(\mathrm{Br} \gamma)$ for differential extinction between nuclear stellar population and Narrow Line Region, in model B. Top, effect on the age derived for the nuclear star cluster. Bottom, effect on the bolometric luminosity derived for the nuclear star cluster. See text about estimates of the maximum 
differential extinction.

Fig. 14. - Effects of correcting the nuclear $\mathrm{EW}(\mathrm{Br} \gamma)$ for asborption of UV and $\mathrm{H} \alpha$ photons in the NLR and other factors possibly responsible for reducing the $L_{L y c}$ derived from the observations (see text), in model B. Top, effect on the age derived for the nuclear star cluster. Bottom, effect on the ratio between the bolometric luminosity derived for the nuclear star cluster and the bolometric luminosity of the AGN. See text about estimates of the maximum $\mathrm{L}_{\text {Lyc }}$ correction. 
Table 1. Nuclear emission lines $\left(\right.$ aperture $\left.=0.75^{\prime \prime}\right)$

\begin{tabular}{lcc}
\hline \hline \multicolumn{1}{c}{ Line } & $\begin{array}{c}\lambda^{a} \\
(\mu \mathrm{m})\end{array}$ & $\begin{array}{c}\text { Flux }^{b} \\
\left(10^{-15} \mathrm{erg} \mathrm{cm}^{-2} \mathrm{~s}^{-1}\right)\end{array}$ \\
& & \\
\hline $\mathrm{H}_{2}(1-0) \mathrm{S}(3)$ & 1.9576 & $14.4 \pm 5$ \\
{$[\mathrm{SiVI}]$} & 1.9635 & $70.2 \pm 5$ \\
$\mathrm{H}_{2}(1-0) \mathrm{S}(2)$ & 2.0332 & $6.09 \pm 1.2$ \\
{$[\mathrm{AlIX}]$} & 2.043 & $15.6 \pm 1.3$ \\
$\mathrm{HeI}$ & 2.0581 & $5.5 \pm 0.7$ \\
$\mathrm{H}_{2}(1-0) \mathrm{S}(1)$ & 2.1218 & $11.5 \pm 0.6$ \\
$\mathrm{Br} \gamma$ & 2.1655 & $15.5 \pm 0.6$ \\
$\mathrm{HeII}$ & 2.1885 & $2.4 \pm 0.6$ \\
$\mathrm{H}_{2}(1-0) \mathrm{S}(0)$ & 2.2235 & $2.07 \pm 0.46$ \\
$\mathrm{H}_{2}(2-1) \mathrm{S}(1)$ & 2.2471 & $1.13 \pm 0.37$ \\
{$[\mathrm{CaVIII}]$} & 2.3213 & $47.5 \pm 5$ \\
$\mathrm{H}_{2}(1-0) \mathrm{Q}(1)$ & 2.4066 & $9.5 \pm 1.9$ \\
\hline
\end{tabular}

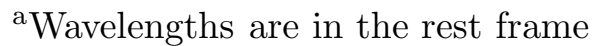

${ }^{\mathrm{b}}$ Fluxes are corrected for a Galactic extinction of $A_{V}=1.5 \mathrm{mag}$, but uncorrected for internal reddenning. 
Table 2. Luminosities and sizes of the nuclear non stellar source at various wavelengths in the near-to-mid IR spectral region.

\begin{tabular}{lcc}
\hline \hline$\lambda(\mu \mathrm{m})$ & $\nu \mathrm{L}_{\nu}\left(\times 10^{7} L_{\odot}\right)$ & Radius $(\mathrm{pc})$ \\
& & \\
\hline $2.2(\mathrm{~K})^{a}$ & 1.5 & $<1.5$ \\
$3.8\left(\mathrm{~L}^{\prime}\right)^{b}$ & 30 & 3 \\
$4.8(\mathrm{M})^{b}$ & 56 & 3 \\
$10.3(\mathrm{~N})^{b}$ & 90 & 13 \\
\hline
\end{tabular}

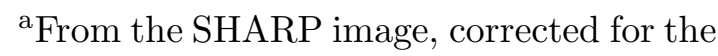
contribution of the stellar light as derived from the stellar CO map (Fig.6)

${ }^{\mathrm{b}}$ From Siebenmorgen et al. (1997).

Table 3. Starburst models and luminosity budget of the various sources in Circinus.

\begin{tabular}{lccc}
\hline \hline \multicolumn{1}{c}{ Source } & Luminosity & Age & Burst duration \\
& & & \\
\hline Observed $^{a}$ & $1.7 \times 10^{10} L_{\odot}$ & & \\
AGN intrinsic $^{b}$ & $10^{10} L_{\odot}$ & & \\
Starburst $(\mathrm{R}<200 \mathrm{pc})$ & $1.1 \times 10^{10} L_{\odot}$ & $10^{8}$ yrs & $10^{7}$ yrs \\
Starburst $(\mathrm{R}<6 \mathrm{pc})$, mod. A & $2 \times 10^{8} L_{\odot}$ & $7 \times 10^{7}$ yrs & $10^{7}$ yrs \\
Starburst $(\mathrm{R}<6 \mathrm{pc})$, mod. B & $2 \times 10^{9} L_{\odot}$ & $5 \times 10^{6}$ yrs & $10^{6}$ yrs \\
\hline
\end{tabular}

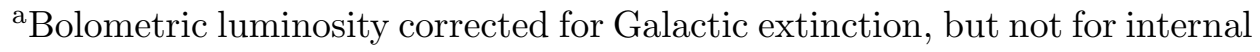
reddening (see note 10 ).

${ }^{\mathrm{b}}$ From Moorwood et al. (1996a). 

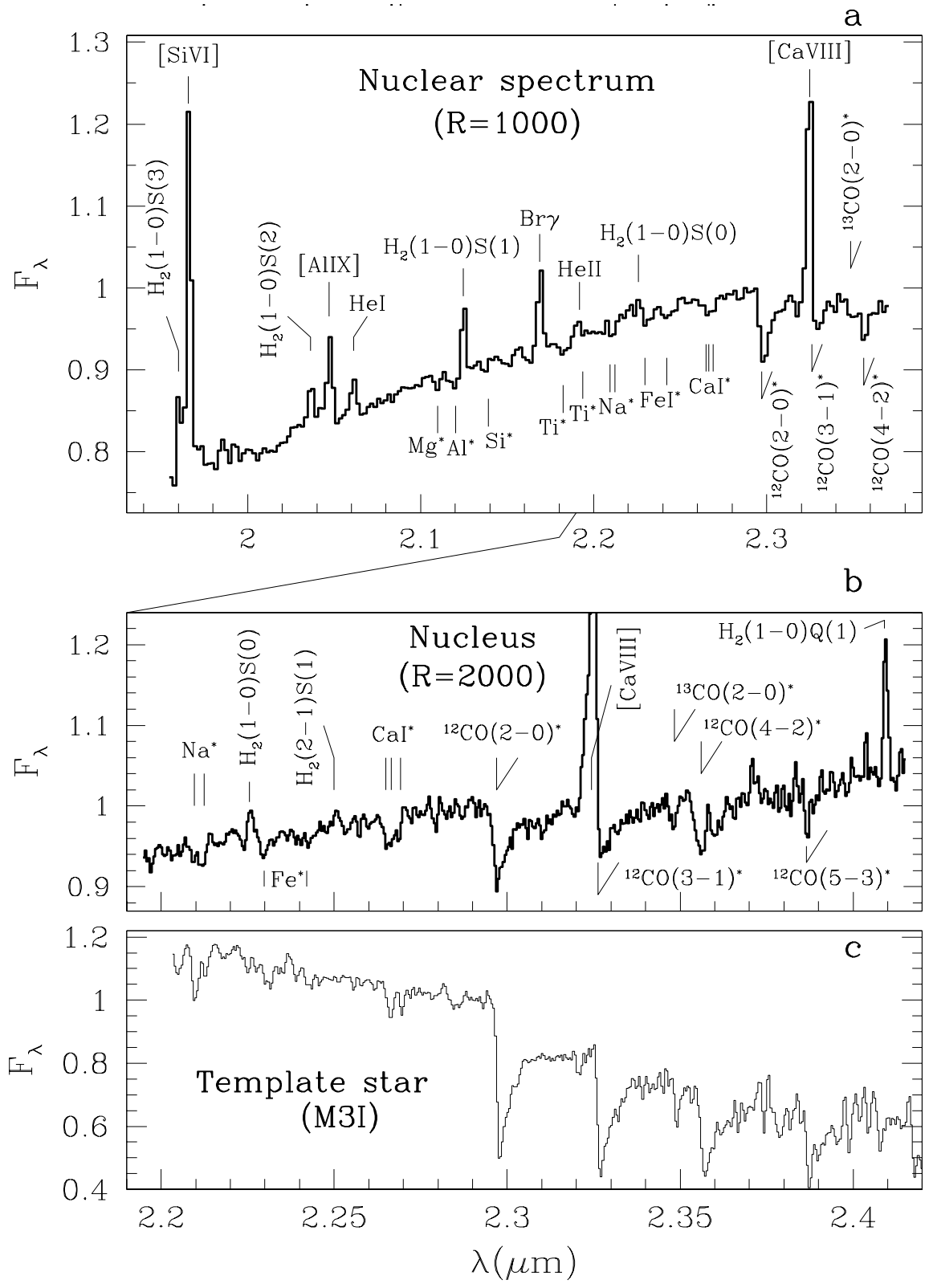

Fig. 3.- 


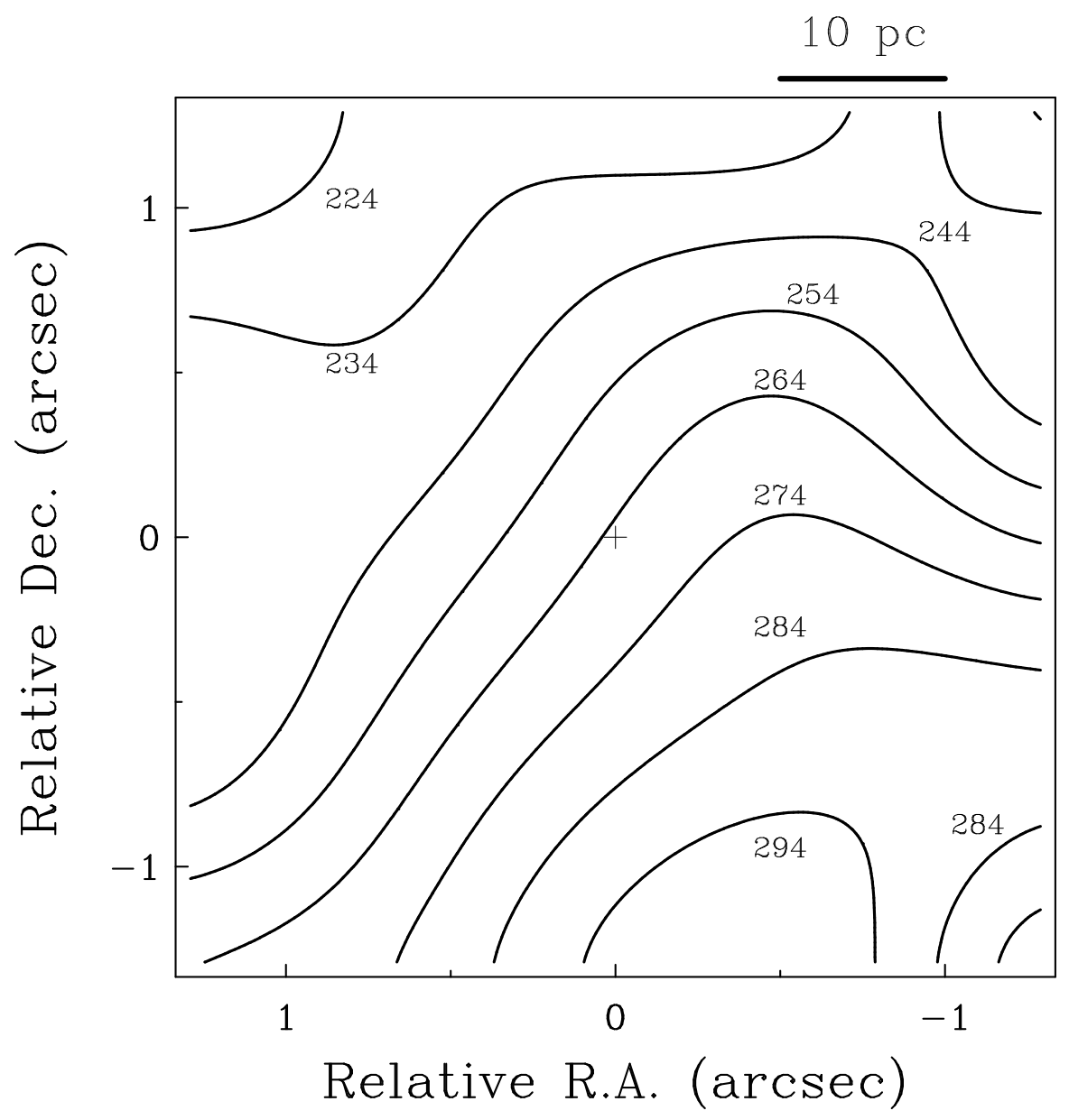

Fig. 5.- 

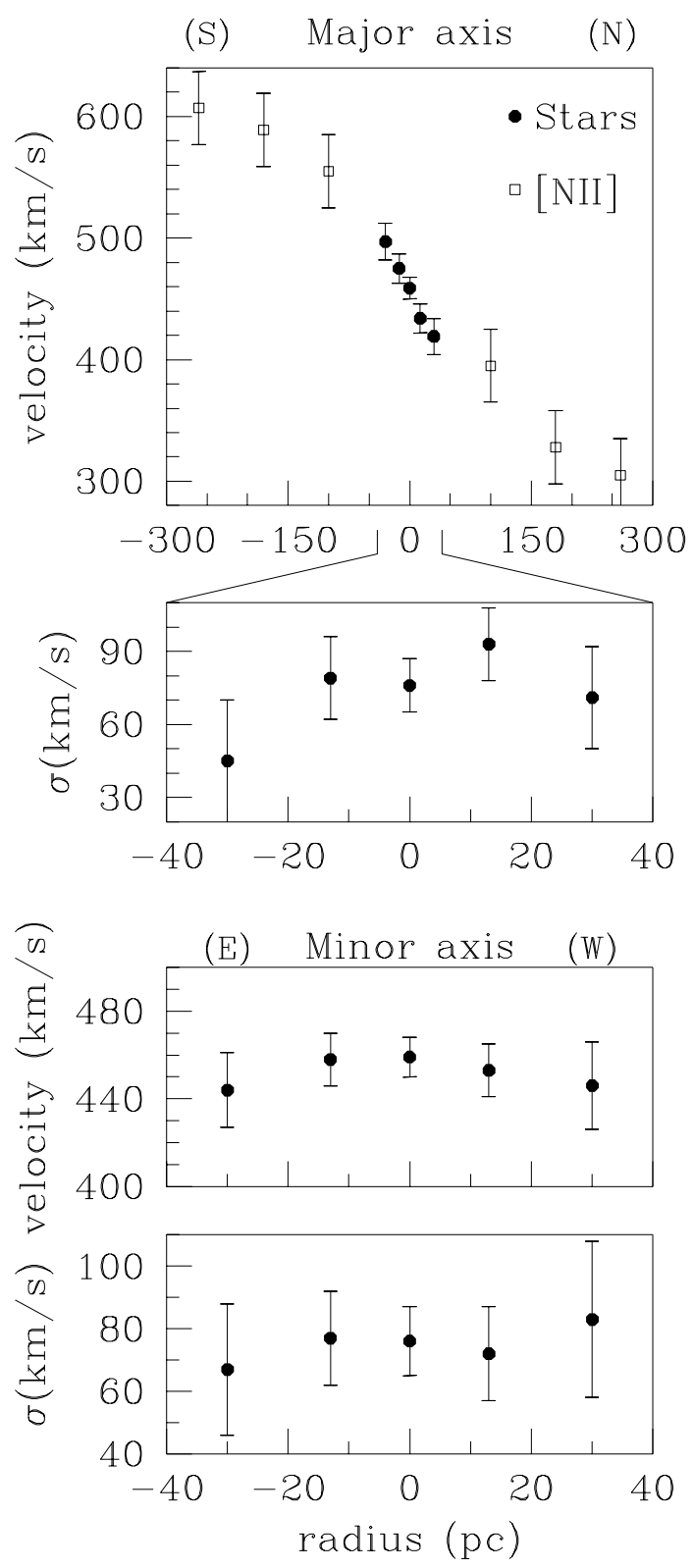

Fig. 6.- 


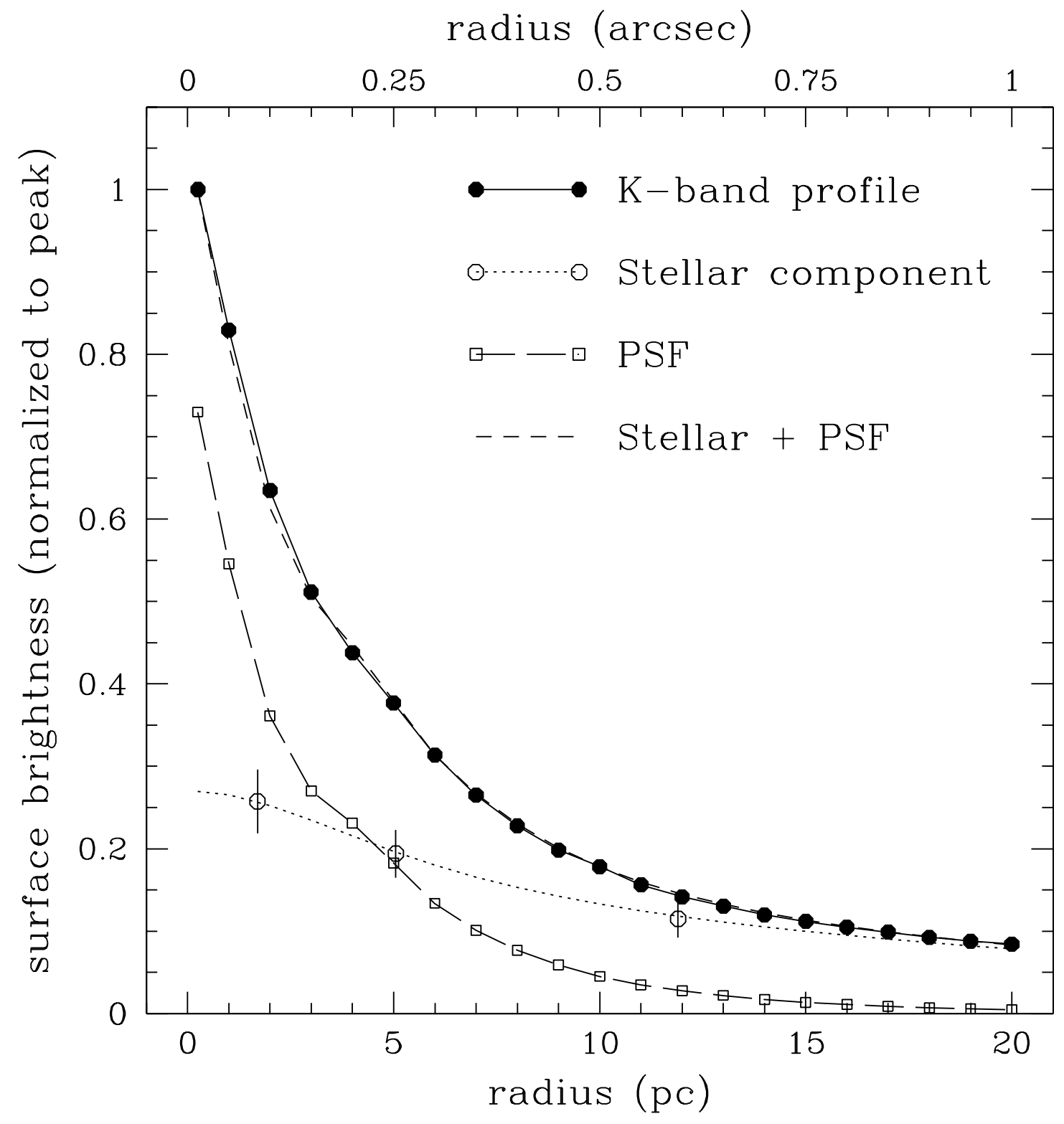

Fig. 7.- 


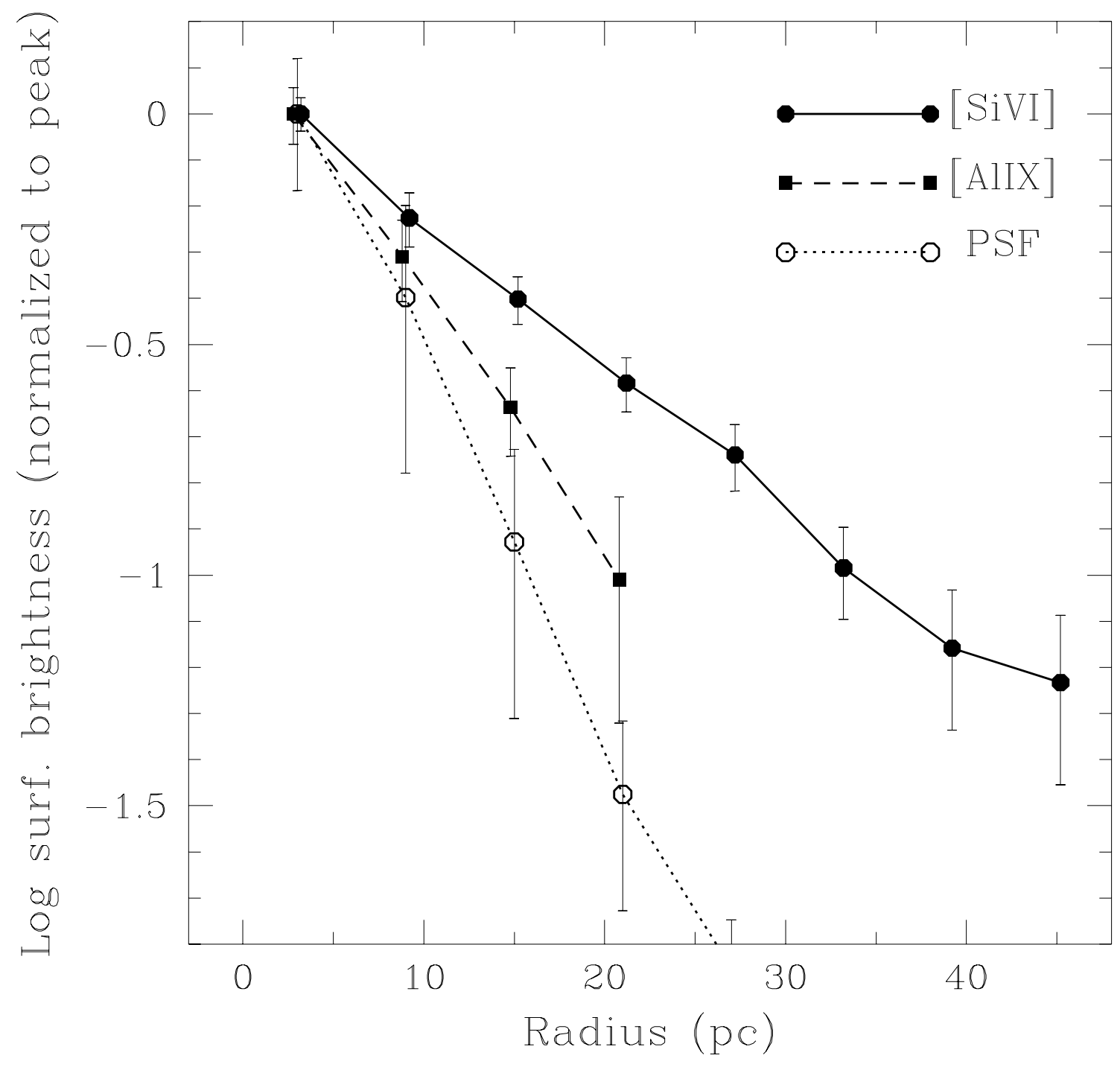

Fig. 8.- 


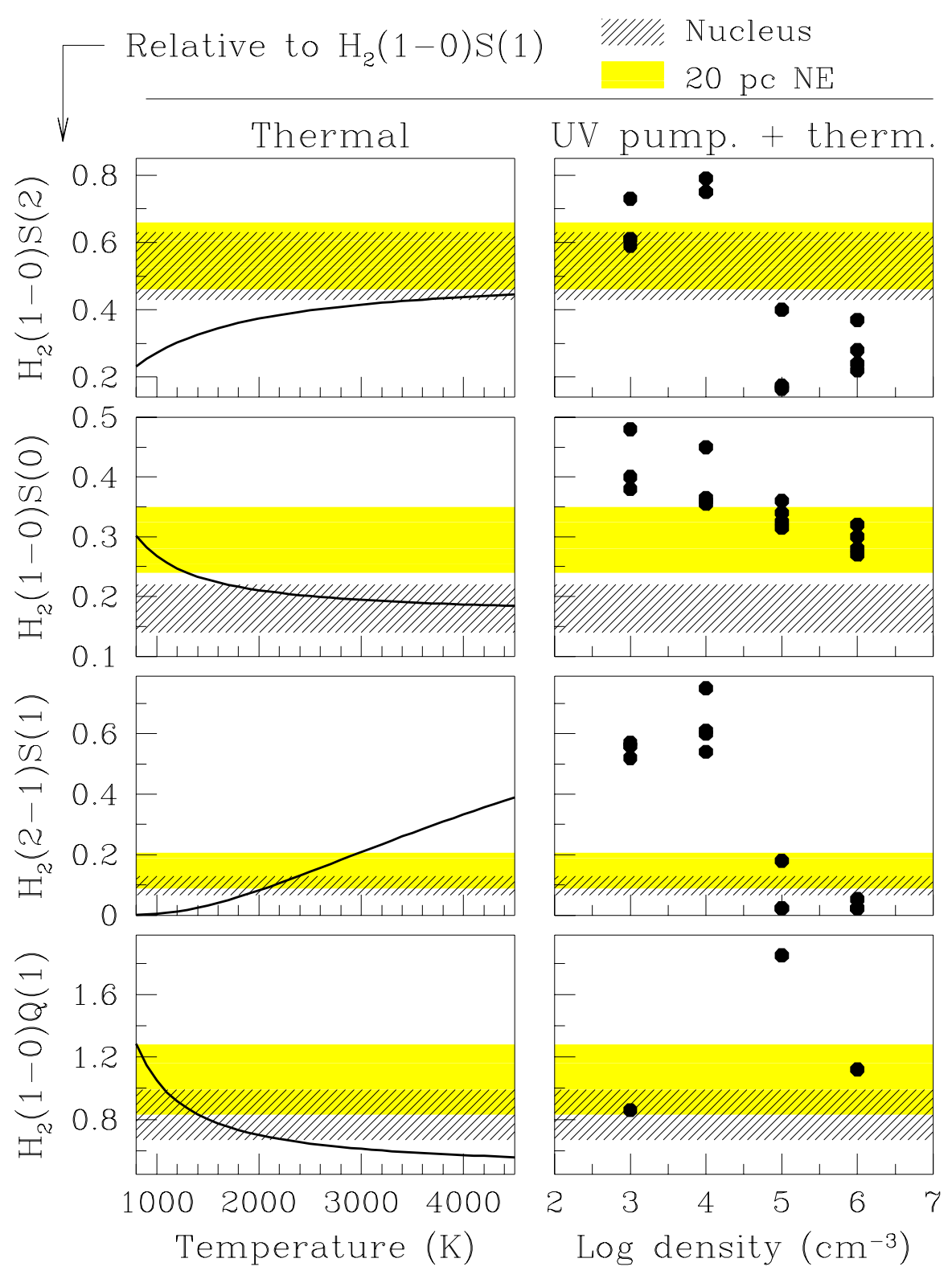

Fig. 9.- 


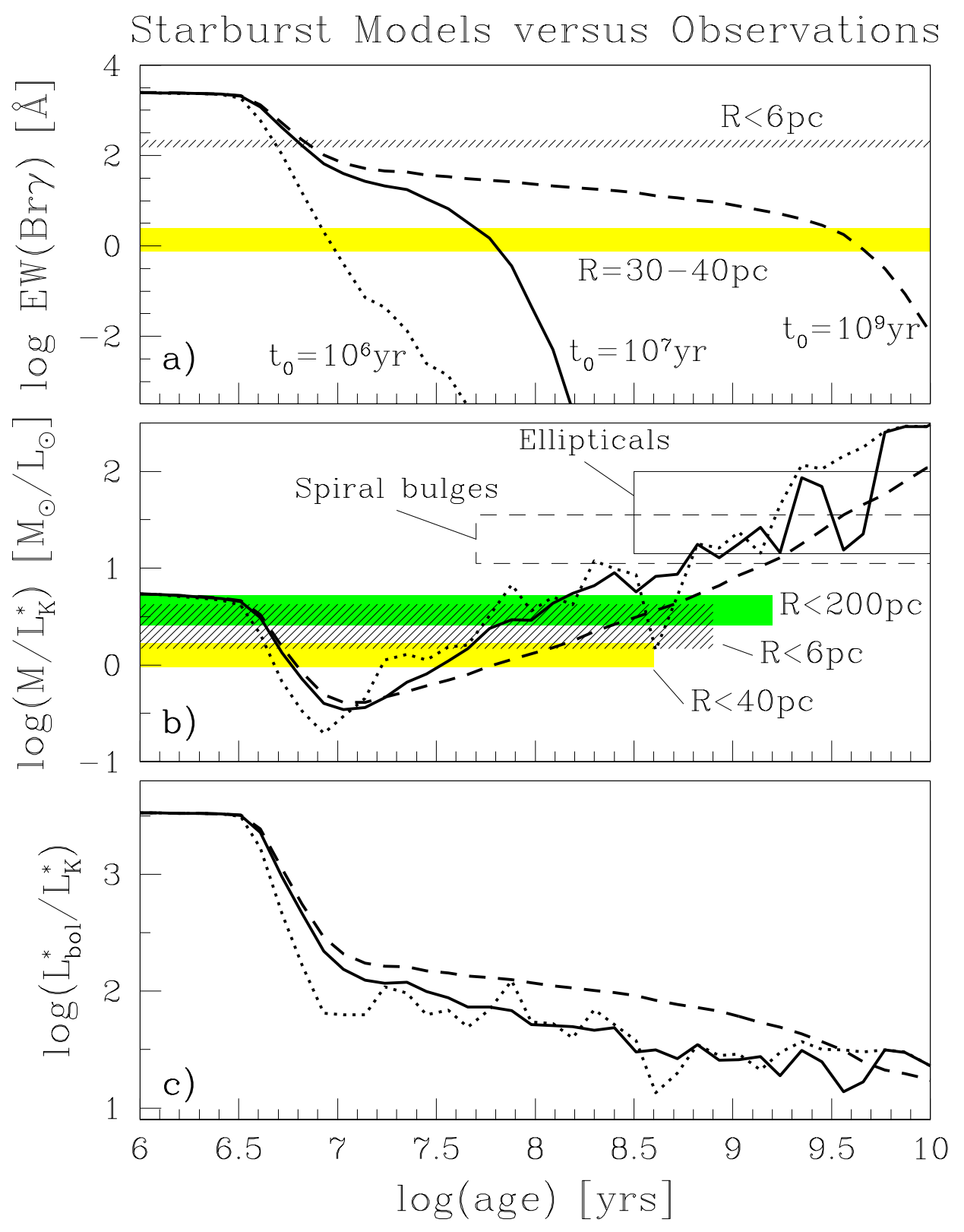

Fig. 10.- 


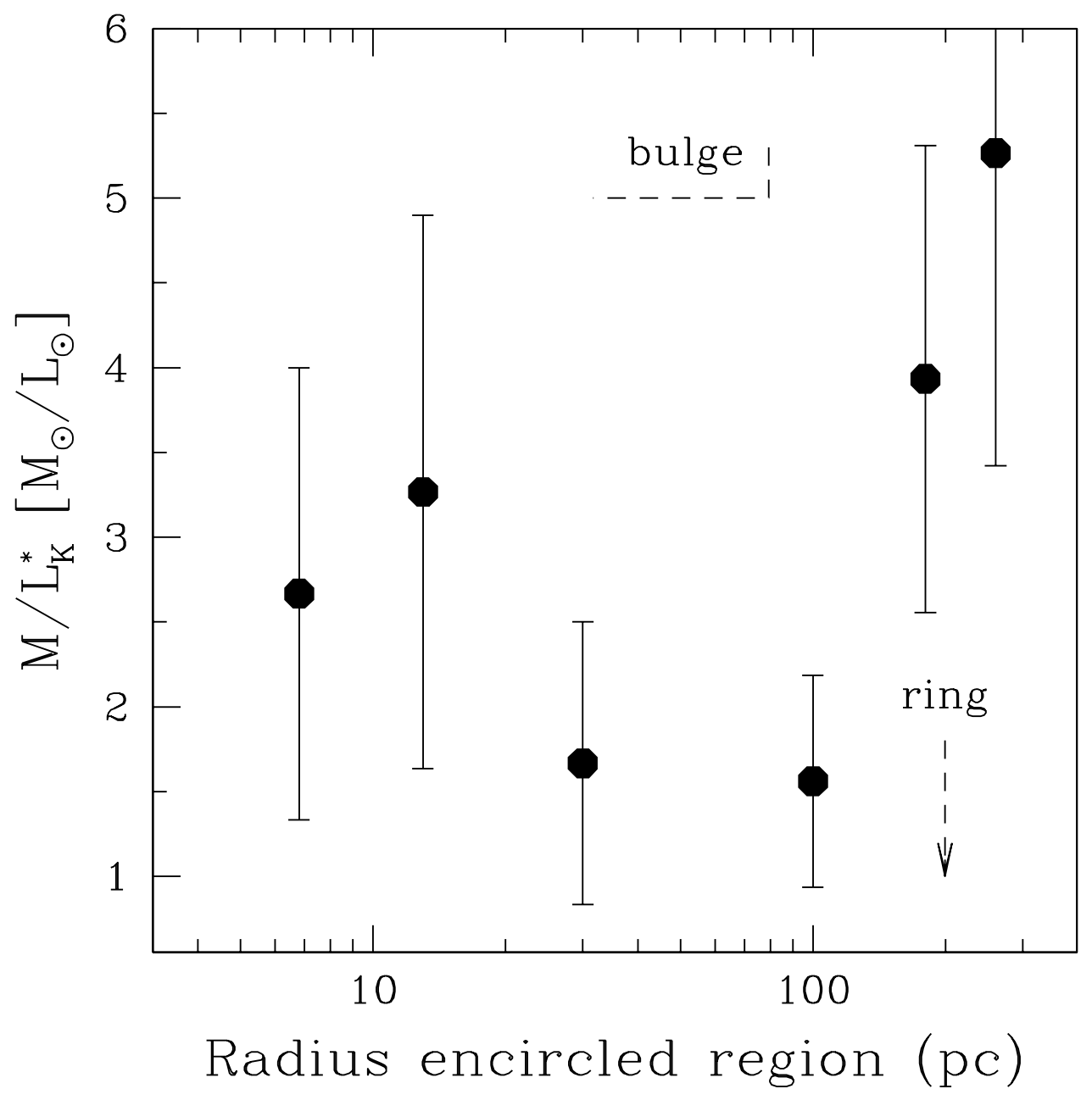

Fig. 11.- 
Effect of excess nuclear extinction (model A)
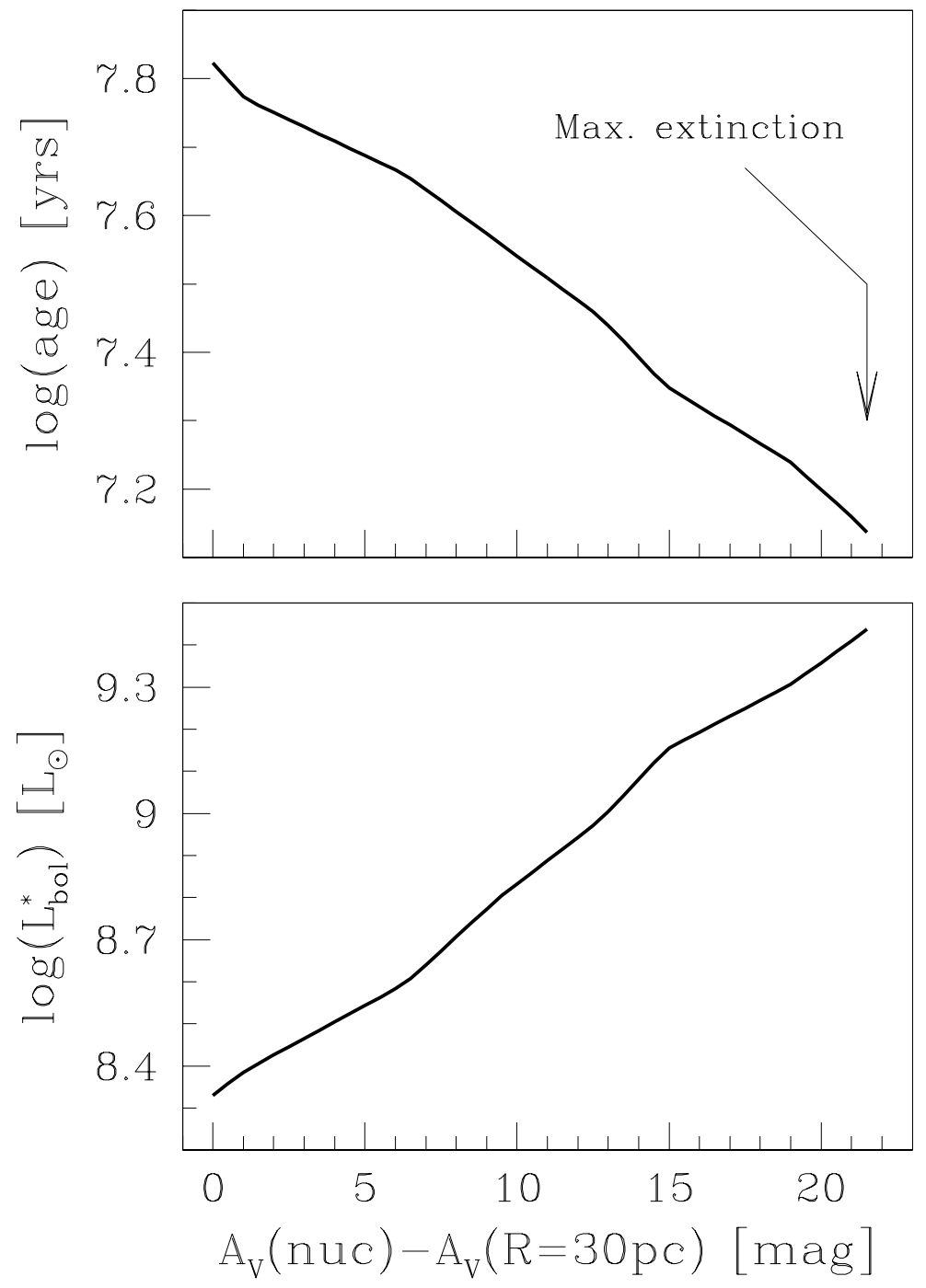

Fig. 12.- 

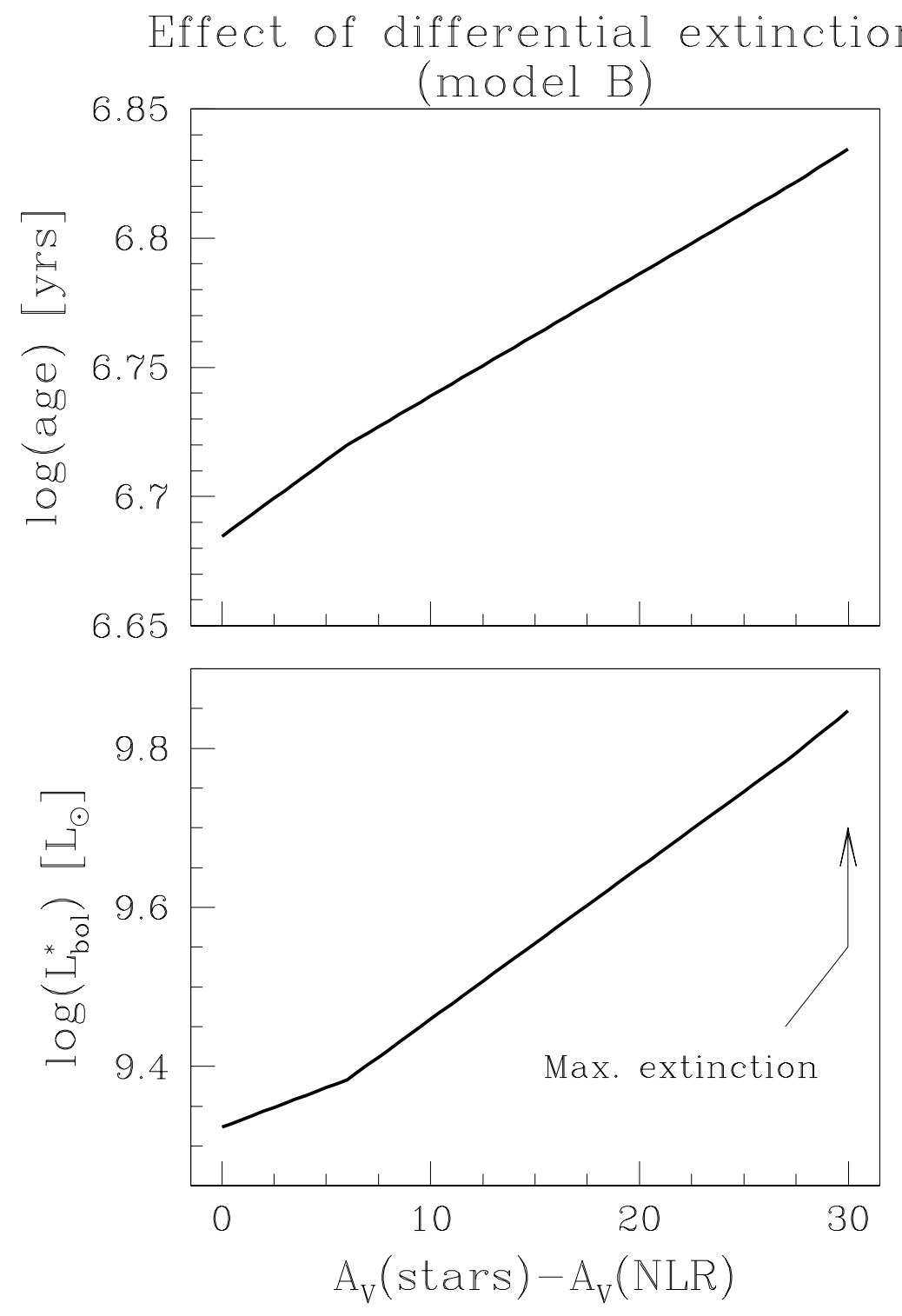

Fig. 13.- 

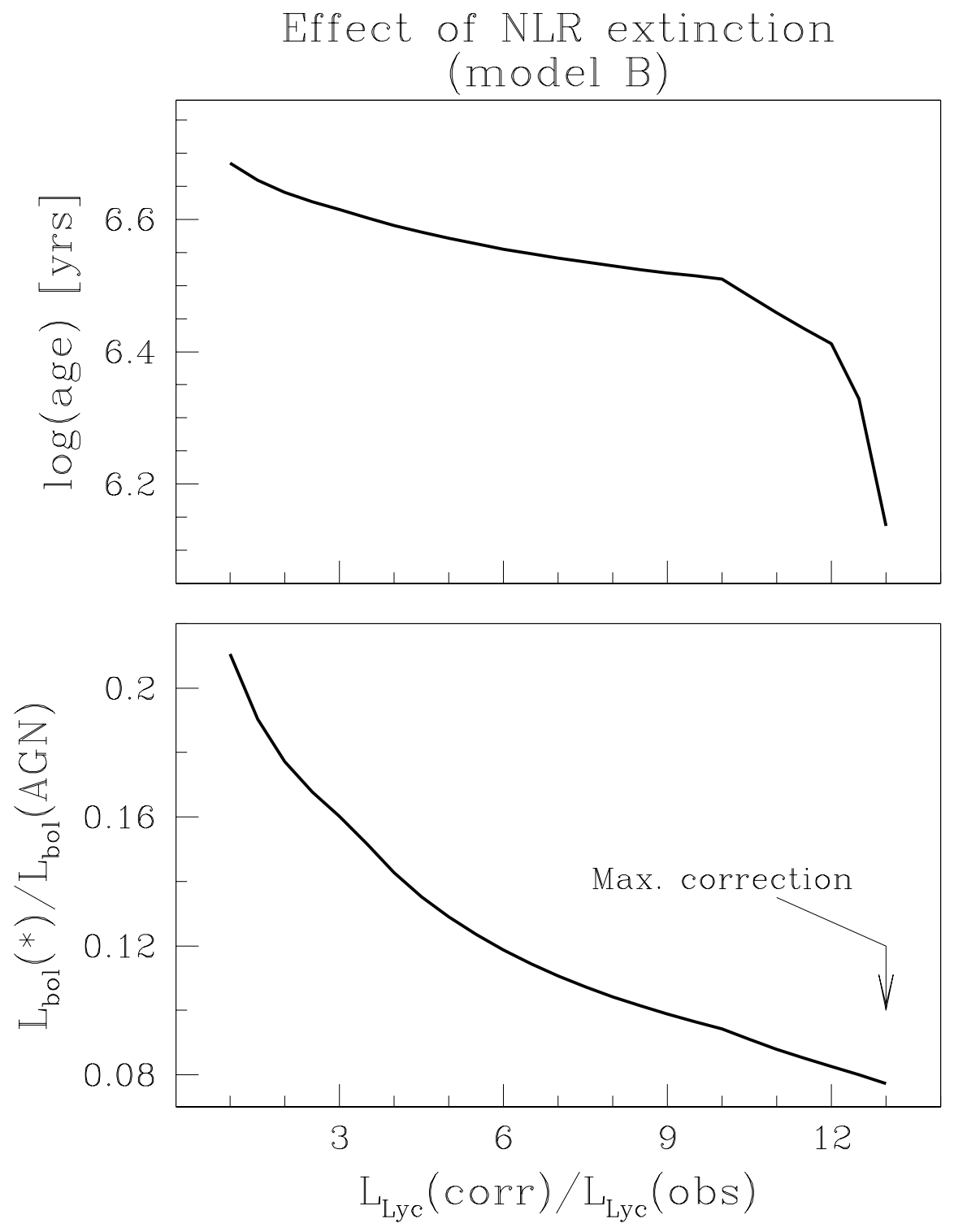

Fig. 14.- 